A. Klar, J. Maringer, R. Wegener

A 3d model for fiber lay-down in nonwoven production processes 
(C) Fraunhofer-Institut für Techno- und Wirtschaftsmathematik ITWM 2010

ISSN 1434-9973

Bericht 198 (2010)

Alle Rechte vorbehalten. Ohne ausdrückliche schriftliche Genehmigung des Herausgebers ist es nicht gestattet, das Buch oder Teile daraus in irgendeiner Form durch Fotokopie, Mikrofilm oder andere Verfahren zu reproduzieren oder in eine für Maschinen, insbesondere Datenverarbeitungsanlagen, verwendbare Sprache zu übertragen. Dasselbe gilt für das Recht der öffentlichen Wiedergabe.

Warennamen werden ohne Gewährleistung der freien Verwendbarkeit benutzt.

Die Veröffentlichungen in der Berichtsreihe des Fraunhofer ITWM können bezogen werden über:

Fraunhofer-Institut für Techno- und Wirtschaftsmathematik ITWM Fraunhofer-Platz 1

67663 Kaiserslautern

Germany

Telefon: $\quad+49(0) 631 / 31600-0$

Telefax: $\quad+49(0) 631 / 31600-1099$

E-Mail: info@itwm.fraunhofer.de

Internet: www.itwm.fraunhofer.de 
Das Tätigkeitsfeld des Fraunhofer-Instituts für Techno- und Wirtschaftsmathematik ITWM umfasst anwendungsnahe Grundlagenforschung, angewandte Forschung sowie Beratung und kundenspezifische Lösungen auf allen Gebieten, die für Techno- und Wirtschaftsmathematik bedeutsam sind.

In der Reihe »Berichte des Fraunhofer ITWM « soll die Arbeit des Instituts kontinuierlich einer interessierten Öffentlichkeit in Industrie, Wirtschaft und Wissenschaft vorgestellt werden. Durch die enge Verzahnung mit dem Fachbereich Mathematik der Universität Kaiserslautern sowie durch zahlreiche Kooperationen mit internationalen Institutionen und Hochschulen in den Bereichen Ausbildung und Forschung ist ein großes Potenzial für Forschungsberichte vorhanden. In die Berichtreihe werden sowohl hervorragende Diplom- und Projektarbeiten und Dissertationen als auch Forschungsberichte der Institutsmitarbeiter und Institutsgäste zu aktuellen Fragen der Techno- und Wirtschaftsmathematik aufgenommen.

Darüber hinaus bietet die Reihe ein Forum für die Berichterstattung über die zahlreichen Kooperationsprojekte des Instituts mit Partnern aus Industrie und Wirtschaft.

Berichterstattung heißt hier Dokumentation des Transfers aktueller Ergebnisse aus mathematischer Forschungs- und Entwicklungsarbeit in industrielle Anwendungen und Softwareprodukte - und umgekehrt, denn Probleme der Praxis generieren neue interessante mathematische Fragestellungen.

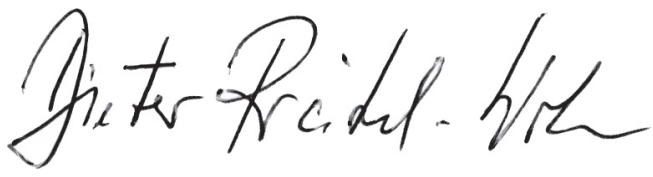

Prof. Dr. Dieter Prätzel-Wolters Institutsleiter

Kaiserslautern, im Juni 2001 



\title{
A 3D MODEL FOR FIBER LAY-DOWN IN NONWOVEN PRODUCTION PROCESSES
}

\author{
A. KLAR ${ }^{\ddagger}$, J. MARINGER $\ddagger$, AND R. WEGENER ${ }^{\ddagger}$
}

\begin{abstract}
In this paper a three dimensional stochastic model for the lay-down of fibers on a moving conveyor belt in the production process of nonwoven materials is derived. The model is based on stochastic differential equations describing the resulting position of the fiber on the belt under the influence of turbulent air flows. The model presented here is an extension of an existing surrogate model, see $[6,3]$.
\end{abstract}

Key words. fiber dynamics, Fokker-Planck equations, diffusion limits

AMS subject classifications. 37H10, 60H30, 41A60, 65C05

1. Introduction. Nonwoven materials are produced in melt-spinning operations: hundreds of individual endless fibers are obtained by the continuous extrusion of a molten polymer through narrow nozzles that are densely and equidistantly placed in a row at a spinning beam. The viscous / viscoelastic fibers are stretched and spun until they solidify due to cooling air streams. Before the elastic fibers lay down on a moving conveyor belt to form a web, they become entangled and form loops due to the highly turbulent air flows. In [12] a general mathematical model for the fiber dynamics is presented which enables the full simulation of the process. Due to the huge amount of physical details these simulations of the fiber spinning and lay-down usually require an extremely large computational effort and high memory storage, see [13]. Thus, a simplified two-dimensional stochastic model for the fiber lay-down process is introduced in [6]. This model describes the position of the fiber on the transport belt by a stochastic differential system containing parameters that characterize the process. These parameters have to be identified from a few representative fibers simulated by the detailed model. Then, the surrogate model can be used to calculate fast and efficiently the behavior of hundreds of long fibers for fleece production. In $[6,7]$ an analytic investigation of the corresponding Fokker-Planck equation has been performed, asymptotic properties and ergodicity of the process have been proven and explicit rates for the convergence to the stationary solution have been obtained.

In the present paper the above $2 \mathrm{D}$ model is extended to three dimensions. This is crucial, if one wants to describe further properties of the resulting web, like permeability. In a first step we revisit the $2 \mathrm{D}$ model and rewrite it in a coordinate free form, see section 2. In section 3 an isotropic 3D model is obtained by a suitable transformation of the deterministic and stochastic processes. Since the resulting fleece is usually rather thin and has an anisotropic orientation of the fibers, we modify the isotropic 3D model accordingly, see section 4 . Further, we investigate the connections between the models by looking at the associated Fokker-Planck equations and their limiting behavior. The limit of large turbulence is considered in section 5 , as well as a large coiling force limit. Finally, we show numerical results and state a possible strategy for parameter estimation of the modified 3D model in section 6 .

\footnotetext{
${ }^{\dagger}$ Fachbereich Mathematik, Technische Universität Kaiserslautern, Germany \{klar@mathematik.uni-kl.de\}

${ }^{\ddagger}$ Fraunhofer ITWM, Kaiserslautern, Germany \{maringer@itwm.fhg.de, wegener@itwm.fhg.de\}
} 
2. Revisiting the 2D Model. Consider a slender, elastic, non-extensible and endless fiber in a lay-down regime. Let the fiber be produced with a certain spinning speed, excited into motion by a surrounding highly turbulent air flow and laid down on a conveyor belt which is for the time being assumed to be non-moving. For the case of a moving belt see Remark 1. Due to its slenderness, the fiber on the two-dimensional transport belt is modeled as an arc-length parametrized curve $\boldsymbol{\xi}: \mathbb{R}_{0}^{+} \rightarrow \mathbb{R}^{2}$, defined by the solution of a dynamical system for $\boldsymbol{\xi}_{t}$ with the arc-length $t$. This dynamical system is given by the following stochastic differential equations, see [6]

$$
\begin{aligned}
d \boldsymbol{\xi}_{t} & =\boldsymbol{\tau}\left(\alpha_{t}\right) d t \\
d \alpha_{t} & =-\nabla V\left(\boldsymbol{\xi}_{t}\right) \cdot \boldsymbol{\tau}^{\perp}\left(\alpha_{t}\right) d t+A d W_{t},
\end{aligned}
$$

equipped with appropriate initial conditions $\boldsymbol{\xi}_{0}, \alpha_{0}$. The normalized tangent is $\boldsymbol{\tau}(\alpha)=$ $(\cos \alpha, \sin \alpha)^{T}$. Introducing the corresponding orthonormal polar unit vector $\boldsymbol{\tau}(\alpha)^{\perp}=$ $(-\sin \alpha, \cos \alpha)^{T}$, the deterministic drift term in the second equation ensures that the fiber tends back to the origin as a consequence of the coiling behavior of the fiber. The throwing ranges of the fiber can be controlled with help of the potential $V$. One may, for example, choose $V(\boldsymbol{\xi})=\left(\xi_{1}^{2} / \sigma_{1}^{2}+\xi_{2}^{2} / \sigma_{2}^{2}\right) / 2$ with the throwing ranges $\sigma_{1}$ and $\sigma_{2}$, see Remark 2. All stochastic effects occurring in the production process are summarized in the one-dimensional Wiener process $\left(W_{t}\right)_{t \geq 0}$ with diffusion constant $A$. This model is refered to as the original $2 D$ model.

We rewrite (2.1) in a coordinate free form using Ito's or Stratonovich's calculus, respectively,

$$
\begin{aligned}
d \boldsymbol{\xi}_{t} & =\boldsymbol{\tau}_{t} d t \\
d \boldsymbol{\tau}_{t} & =-\left(\nabla V\left(\boldsymbol{\xi}_{t}\right) \cdot \boldsymbol{\tau}_{t}^{\perp}\right) \boldsymbol{\tau}_{t}^{\perp} d t-\frac{1}{2} A^{2} \boldsymbol{\tau}_{t} d t+\boldsymbol{\tau}_{t}^{\perp} A d W_{t} \\
& =-\left(\nabla V\left(\boldsymbol{\xi}_{t}\right) \cdot \boldsymbol{\tau}_{t}^{\perp}\right) \boldsymbol{\tau}_{t}^{\perp} d t+\boldsymbol{\tau}_{t}^{\perp} \circ A d W_{t} \\
\left\|\boldsymbol{\tau}_{t}\right\| & =1
\end{aligned}
$$

or in more compact form

$$
\begin{aligned}
d \boldsymbol{\xi}_{t} & =\boldsymbol{\tau}_{t} d t \\
d \boldsymbol{\tau}_{t} & =\left(I-\boldsymbol{\tau}_{t} \otimes \boldsymbol{\tau}_{t}\right)\left(-\nabla V\left(\boldsymbol{\xi}_{t}\right) d t+A d \boldsymbol{W}_{t}\right)-\frac{1}{2} A^{2} \boldsymbol{\tau}_{t} d t \\
& =\left(I-\boldsymbol{\tau}_{t} \otimes \boldsymbol{\tau}_{t}\right) \circ\left(-\nabla V\left(\boldsymbol{\xi}_{t}\right) d t+A d \boldsymbol{W}_{t}\right) \\
\left\|\boldsymbol{\tau}_{t}\right\| & =1
\end{aligned}
$$

Thereby, $I$ denotes the identity matrix, o denotes the usage of Stratonovich integrals and $\left(\boldsymbol{W}_{t}\right)_{t \geq 0}$ is a two-dimensional Wiener process. The algebraic constraint takes into account the arc-length parametrization for the inextensible fibers. We note that the stochastic part in (2.2) is a Brownian motion on the unit circle, see for example [14].

REMARK 1. Let the fiber be produced with the spinning speed $v_{\text {spin }}$ and laid down on a conveyor belt moving with the velocity $v_{\text {belt }}$. The fiber curve is now denoted as $\boldsymbol{\eta}: \mathbb{R}_{0}^{+} \rightarrow \mathbb{R}^{2}$. The arc-length parametrization and in-extensibility gives again

$$
d \boldsymbol{\eta}_{t}=\left(\begin{array}{c}
\cos \alpha_{t} \\
\sin \alpha_{t}
\end{array}\right) d t
$$


where $\alpha$ denotes the angle of the fiber relative to the direction of motion $\boldsymbol{e}_{1}$ of the transport belt. The reference point of the spinning process determined by the position of the nozzle moves in the coordinate system of the transport belt in the direction $-\boldsymbol{e}_{1}$. Thus,

$$
\boldsymbol{\xi}(t)=\boldsymbol{\eta}(t)-\left(-\kappa t \boldsymbol{e}_{1}\right)
$$

describes the deviation of the fiber from the reference point as a function of the arclength parameter $t$, where $\kappa=v_{\text {belt }} / v_{\text {spin }} \in[0,1]$ is the ratio between the belt and spinning speeds. Following [6], we model $(\boldsymbol{\xi}, \alpha)$ by the stochastic differential system

$$
\begin{aligned}
d \boldsymbol{\xi}_{t} & =\boldsymbol{\tau}\left(\alpha_{t}\right) d t+\kappa \boldsymbol{e}_{1} d t \\
d \alpha_{t} & =-\nabla V\left(\boldsymbol{\xi}_{t}\right) \cdot \boldsymbol{\tau}_{t}^{\perp} d t+A d W_{t} .
\end{aligned}
$$

We might generalize the last model with help of the concept of reference curves allowing the consideration of further specific production processes, see [10]. Introducing a reference curve $\boldsymbol{\gamma}$ the generalized model reads

$$
\begin{aligned}
d \boldsymbol{\xi}_{t} & =\boldsymbol{\tau}\left(\alpha_{t}\right) d t+d \boldsymbol{\gamma}_{t} \\
d \alpha_{t} & =-\nabla V\left(\boldsymbol{\xi}_{t}\right) \cdot \boldsymbol{\tau}_{t}^{\perp} d t+A d W_{t} .
\end{aligned}
$$

REMARK 2. The Fokker-Planck equation associated to (2.1) reads

$$
\partial_{t} P=-\boldsymbol{\tau} \cdot \nabla_{\xi} P+\nabla V(\boldsymbol{\xi}) \cdot \partial_{\alpha}\left(\boldsymbol{\tau}^{\perp} P\right)+\frac{1}{2} A^{2} \partial_{\alpha \alpha} P
$$

and the stationary density is given by

$$
P_{\text {stat }}(\boldsymbol{\xi})=C \exp (-V(\boldsymbol{\xi})),
$$

where $C$ is a normalization constant. Using the potential $V(\boldsymbol{\xi})=\left(\xi_{1}^{2} / \sigma_{1}^{2}+\xi_{2}^{2} / \sigma_{2}^{2}\right) / 2$ the throwing ranges $\sigma_{1}, \sigma_{2}$ are interpreted as standard deviations $\sigma_{1}, \sigma_{2}$ of the normal distribution.

REMARK 3. The limit for large values of the diffusion coefficient $A$ is

$$
d \boldsymbol{\xi}_{t}=-\frac{1}{A^{2}} \nabla V\left(\boldsymbol{\xi}_{t}\right) d t+\sqrt{2} \frac{1}{A} d \boldsymbol{W}_{t},
$$

see [3]. It will be called the reduced 2D model in the following.

3. The Isotropic 3D Model. In this and the following section the above described two-dimensional model will be extended to three dimensions. Again, we model the motion of the fiber as an arc-length parametrized curve $\boldsymbol{\xi}: \mathbb{R}_{0}^{+} \rightarrow \mathbb{R}^{3}$ with normalized tangent $\boldsymbol{\tau}$, which is in spherical coordinates given by

$$
\boldsymbol{\tau}(\alpha, \theta)=\left(\begin{array}{c}
\cos \alpha \sin \theta \\
\sin \alpha \sin \theta \\
\cos \theta
\end{array}\right) .
$$


We introduce the orthonormal spherical unit vectors

$$
\boldsymbol{n}_{1}(\alpha)=\left(\begin{array}{c}
-\sin \alpha \\
\cos \alpha \\
0
\end{array}\right), \quad \boldsymbol{n}_{2}(\alpha, \theta)=\left(\begin{array}{c}
\cos \alpha \cos \theta \\
\sin \alpha \cos \theta \\
-\sin \theta
\end{array}\right)
$$

and follow the procedure for the $2 \mathrm{D}$ case to get the stochastic differential equations in local coordinates in the 3D case. We start by translating the Stratonovich coordinate free formulation (2.2) to three dimensions introducing an additional factor $\frac{1}{2}$ :

$$
\begin{aligned}
d \boldsymbol{\xi}_{t} & =\boldsymbol{\tau}_{t} d t \\
d \boldsymbol{\tau}_{t} & =\left(I-\boldsymbol{\tau}_{t} \otimes \boldsymbol{\tau}_{t}\right) \circ\left(-\frac{1}{2} \nabla V\left(\boldsymbol{\xi}_{t}\right) d t+A d \boldsymbol{W}_{t}\right) \\
\left\|\boldsymbol{\tau}_{t}\right\| & =1 .
\end{aligned}
$$

Considering the deterministic part separately gives

$$
\begin{aligned}
d \boldsymbol{\tau}_{t} & =-\frac{1}{2}\left(I-\boldsymbol{\tau}_{t} \otimes \boldsymbol{\tau}_{t}\right) \nabla V\left(\boldsymbol{\xi}_{t}\right) d t \\
& =-\frac{1}{2}\left(\boldsymbol{n}_{1 t} \otimes \boldsymbol{n}_{1 t}+\boldsymbol{n}_{2 t} \otimes \boldsymbol{n}_{2 t}\right) \nabla V\left(\boldsymbol{\xi}_{t}\right) d t \\
& =-\frac{1}{2}\left(\nabla V\left(\boldsymbol{\xi}_{t}\right) \cdot \boldsymbol{n}_{1 t}\right) \boldsymbol{n}_{1 t} d t-\frac{1}{2}\left(\nabla V\left(\boldsymbol{\xi}_{t}\right) \cdot \boldsymbol{n}_{2 t}\right) \boldsymbol{n}_{2 t} d t .
\end{aligned}
$$

Applying the chain rule $(\boldsymbol{\tau}=\boldsymbol{\tau}(\alpha, \theta))$ and comparison of coefficients leads to the deterministic equations in local coordinates

$$
\begin{aligned}
\sin \theta_{t} d \alpha_{t} & =-\frac{1}{2} \nabla V\left(\boldsymbol{\xi}_{t}\right) \cdot \boldsymbol{n}_{1}\left(\alpha_{t}\right) d t \\
d \theta_{t} & =-\frac{1}{2} \nabla V\left(\boldsymbol{\xi}_{t}\right) \cdot \boldsymbol{n}_{2}\left(\alpha_{t}, \theta_{t}\right) d t .
\end{aligned}
$$

Next, we rewrite the stochastic part in Ito's formulation and compute

$$
\begin{aligned}
d \boldsymbol{\tau}_{t} & =\left(I-\boldsymbol{\tau}_{t} \otimes \boldsymbol{\tau}_{t}\right) A d \boldsymbol{W}_{t}-A^{2} \boldsymbol{\tau}_{t} d t \\
& =\left(\boldsymbol{n}_{1 t} \otimes \boldsymbol{n}_{1 t}+\boldsymbol{n}_{2 t} \otimes \boldsymbol{n}_{2 t}\right) A d \boldsymbol{W}_{t}-A^{2} \boldsymbol{\tau}_{t} d t \\
& =\boldsymbol{n}_{1 t} A d W_{t}^{(1)}+\boldsymbol{n}_{2 t} A d W_{t}^{(2)}-A^{2} \boldsymbol{\tau}_{t} d t,
\end{aligned}
$$

which is a Brownian motion on the unit sphere, see [14] or [15]. $\left(W_{t}^{(1)}\right)_{t \geq 0}$ and $\left(W_{t}^{(2)}\right)_{t \geq 0}$ are one-dimensional Wiener processes.

Using Ito's formula for $\boldsymbol{\tau}(\alpha, \theta)$ the stochastic part in local coordinates is given by

$$
\begin{aligned}
\sin \theta_{t} d \alpha_{t} & =A d W_{t}^{(1)} \\
d \theta_{t} & =\frac{1}{2} A^{2} \cot \theta_{t} d t+A d W_{t}^{(2)} .
\end{aligned}
$$

Alltogether the three dimensional model equations in spherical polar coordinates are

$$
\begin{aligned}
d \boldsymbol{\xi}_{t} & =\boldsymbol{\tau}\left(\alpha_{t}, \theta_{t}\right) d t \\
\sin \theta_{t} d \alpha_{t} & =-\frac{1}{2} \nabla V\left(\boldsymbol{\xi}_{t}\right) \cdot \boldsymbol{n}_{1}\left(\alpha_{t}\right) d t+A d W_{t}^{(1)} \\
d \theta_{t} & =-\frac{1}{2} \nabla V\left(\boldsymbol{\xi}_{t}\right) \cdot \boldsymbol{n}_{2}\left(\alpha_{t}, \theta_{t}\right) d t+\frac{1}{2} A^{2} \cot \theta_{t} d t+A d W_{t}^{(2)} .
\end{aligned}
$$



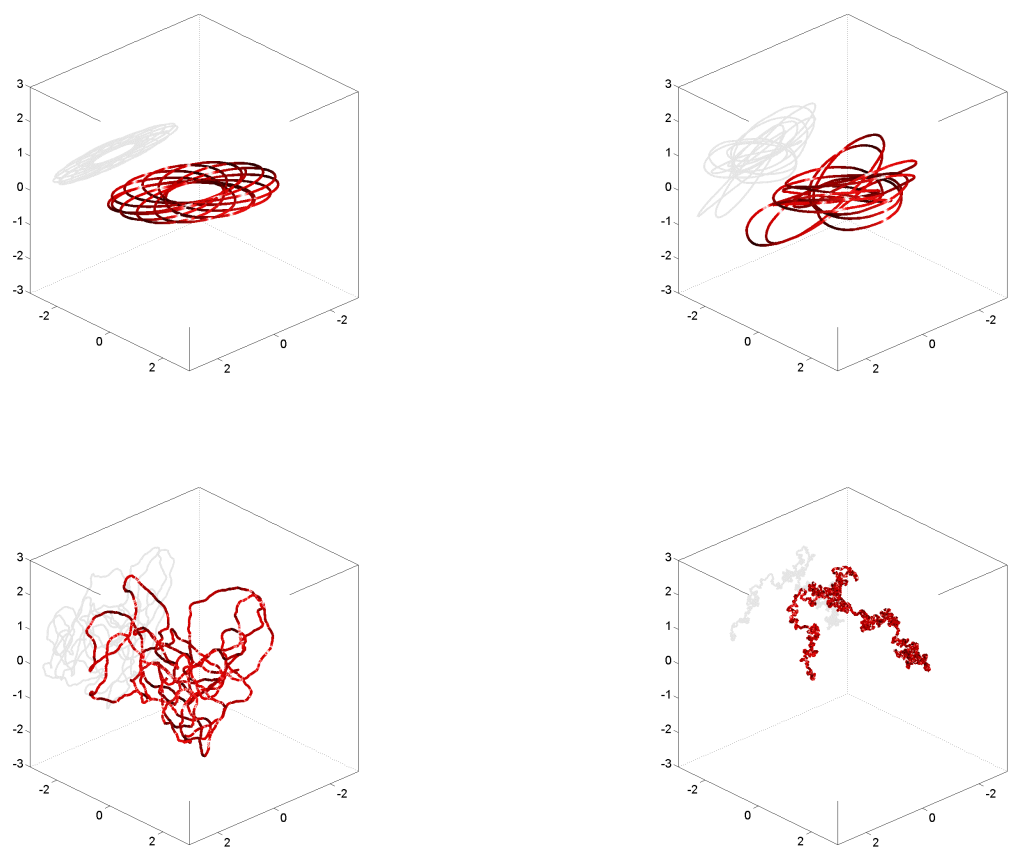

Fig. 3.1: Influence of noise on fiber trajectories. From left to right, top to bottom $A=0 ; 0.1 ; 1 ; 5$.

In the following we refer to this system as the isotropic $3 D$ model. Choosing a symmetric potential $V=V(|\boldsymbol{\xi}|)$ the model is invariant under rotations.

Representative fiber scenarios for varying noise amplitude $A$ are illustrated in Figure 3.1 computed by (3.4) after nondimensionalization using an isotropic potential $V(\boldsymbol{\xi})=|\boldsymbol{\xi}|^{2} / 2$.

However in typical fiber lay-down processes the resulting nonwoven have usually an anisotropic orientation of the fibers as consequence of physical properties and limitations given for example by the impenetrable conveyor belt. For example, the orientation of most of the fibers will concentrate in a direction parallel to the belt. Thus, it is necessary to modify the model such that the orientation of the fibers can be controlled and adapted to measurements. This will be done in the next section.

REMARK 4. The generalization of the model using arbitrary reference curves $\gamma$ is obviously

$$
\begin{aligned}
d \boldsymbol{\xi}_{t} & =\boldsymbol{\tau}\left(\alpha_{t}, \theta_{t}\right) d t+d \boldsymbol{\gamma}_{t} \\
\sin \theta_{t} d \alpha_{t} & =-\frac{1}{2} \nabla V\left(\boldsymbol{\xi}_{t}\right) \cdot \boldsymbol{n}_{1}\left(\alpha_{t}\right) d t+A d W_{t}^{(1)} \\
d \theta_{t} & =-\frac{1}{2} \nabla V\left(\boldsymbol{\xi}_{t}\right) \cdot \boldsymbol{n}_{2}\left(\alpha_{t}, \theta_{t}\right) d t+\frac{1}{2} A^{2} \cot \theta_{t} d t+A d W_{t}^{(2)} .
\end{aligned}
$$


REMARK 5. The Fokker-Planck equation associated to the isotropic model with nonmoving belt is

$$
\begin{aligned}
\partial_{t} P= & -\boldsymbol{\tau} \cdot \nabla_{\xi} P+\frac{1}{\sin \theta} \frac{1}{2} \nabla V(\boldsymbol{\xi}) \cdot \partial_{\alpha}\left(\boldsymbol{n}_{1} P\right)+\frac{1}{2} \nabla V(\boldsymbol{\xi}) \cdot \partial_{\theta}\left(\boldsymbol{n}_{2} P\right) \\
& -\frac{1}{2} A^{2} \partial_{\theta}(\cot \theta P)+\frac{1}{2} \frac{A^{2}}{\sin ^{2} \theta} \partial_{\alpha \alpha} P+\frac{1}{2} A^{2} \partial_{\theta \theta} P
\end{aligned}
$$

and the stationary density is given by

$$
P_{\text {stat }}(\boldsymbol{\xi}, \theta)=C \sin \theta \exp (-V(\boldsymbol{\xi})) .
$$

4. The Modified 3D Model. The idea of the modified model is that by introducing an additional parameter $B$ we obtain a $3 \mathrm{D}$ model which can be adapted to the distributions of the $\theta$-angle in a realistic fleece. With the help of this parameter we are able to weight the directions of the spherical unit-vectors $\boldsymbol{n}_{1}$ and $\boldsymbol{n}_{2}$ differently, such that it is possible to capture the anisotropic orientation of the fibers. We suppose that the belt lies in the $\left(\boldsymbol{e}_{1}, \boldsymbol{e}_{2}\right)$-plane and that the spherical coordinates are determined in a standard way, that means $\theta$ is the angle between the direction $\boldsymbol{e}_{3}$ and the tangent $\tau$ on the fiber, whereas $\alpha$ is the angle between the direction $\boldsymbol{e}_{1}$ and the projection of $\tau$ on the reference plane.

Let $B \in[0,1]$, then we replace in (3.2) the factor $\frac{1}{2}$ as follows

$$
d \boldsymbol{\tau}_{t}=-\frac{1}{B+1}\left(\left(\nabla V\left(\boldsymbol{\xi}_{t}\right) \cdot \boldsymbol{n}_{1 t}\right) \boldsymbol{n}_{1 t}+B\left(\nabla V\left(\boldsymbol{\xi}_{t}\right) \cdot \boldsymbol{n}_{2 t}\right) \boldsymbol{n}_{2 t}\right) d t .
$$

We note, that if $B=0$ then we recover the deterministic part in the 2D case observing that $\boldsymbol{n}_{1}$ is the $3 \mathrm{D}$ analogue of $\boldsymbol{\tau}^{\perp}$. If $B=1$ the isotropic 3 -D model is recovered. Furthermore, we change the stochastic part (3.3) redefining

$$
d \boldsymbol{\tau}_{t}=\boldsymbol{n}_{1 t} A d W_{t}^{(1)}+\sqrt{B} \boldsymbol{n}_{2 t} A d W_{t}^{(2)}-\frac{1}{2}(1+B) A^{2} \boldsymbol{\tau}_{t} d t .
$$

This ensures again, that for $B=0$ we are back to the $2 \mathrm{D}$ case and for $B=1$ to the isotropic $3 \mathrm{D}$ case. The modified $3 \mathrm{D}$ model ranges between the isotropic $3 \mathrm{D}$ model and the 2D model describing fibers with an orientation ranging between a random orientation and an orientation in the $\left(\boldsymbol{e}_{1}, \boldsymbol{e}_{2}\right)$-plane. Using Ito's formula, we obtain the modified 3D model in local coordinates

$$
\begin{aligned}
d \boldsymbol{\xi}_{t} & =\boldsymbol{\tau}\left(\alpha_{t}, \theta_{t}\right) d t \\
\sin \theta_{t} d \alpha_{t} & =-\frac{1}{B+1} \nabla V\left(\boldsymbol{\xi}_{t}\right) \cdot \boldsymbol{n}_{1}\left(\alpha_{t}\right) d t+A d W_{t}^{(1)} \\
d \theta_{t} & =-\frac{B}{B+1} \nabla V\left(\boldsymbol{\xi}_{t}\right) \cdot \boldsymbol{n}_{2}\left(\alpha_{t}, \theta_{t}\right) d t+\frac{1}{2} A^{2} \cot \theta_{t} d t+\sqrt{B} A d W_{t}^{(2)} .
\end{aligned}
$$

The effect of varying parameter $B$ in (4.3) for an isotropic potential $V(\boldsymbol{\xi})=|\boldsymbol{\xi}|^{2} / 2$ is shown in Figure 4.1. The noise amplitude is chosen as $A=1$. One observes that for decreasing $B$ the orientation of the fibers is getting more anisotropic. This corresponds to a distribution of the angle $\theta$ concentrating around $\theta=\pi / 2$. 

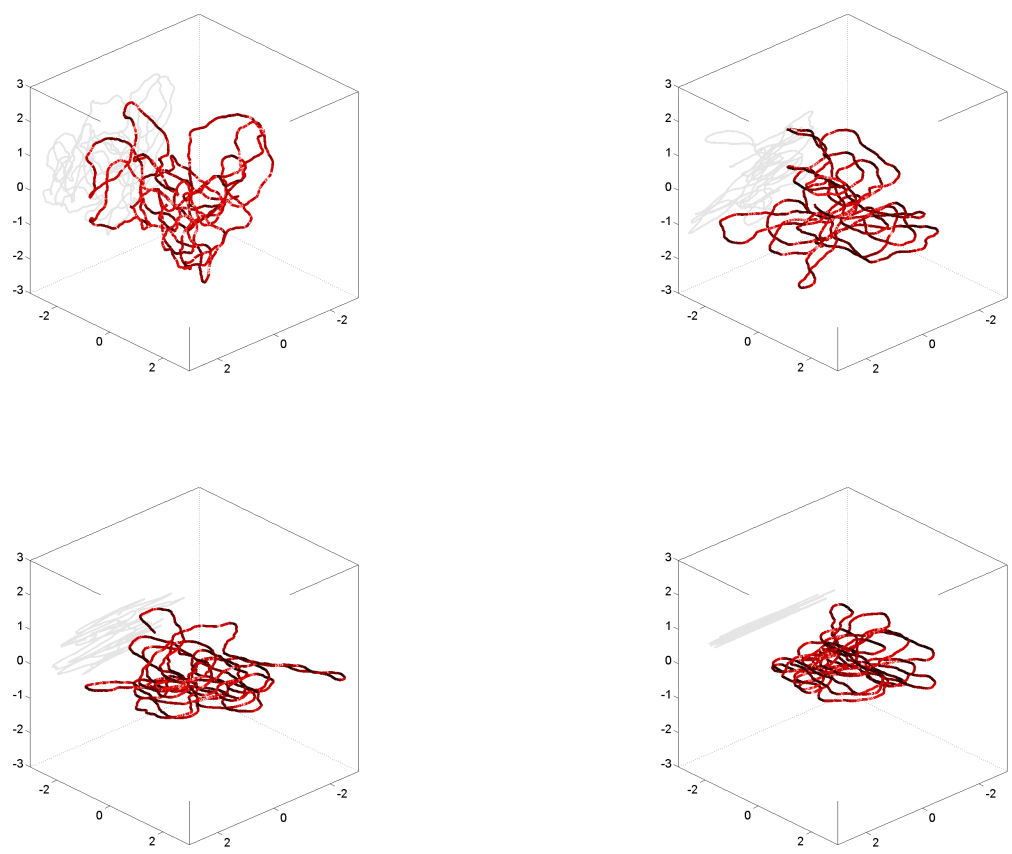

Fig. 4.1: Influence of the parameter $B$ on fiber trajectories. From left to right, top to bottom $B=1 ; 0.1 ; 0.01 ; 0.0001$.

5. Asymptotic Limits and Connections between the Models. In the previous chapters we have distinguished between the $2 \mathrm{D}$ model $(2.1)$, the isotropic $3 \mathrm{D}$ model (3.4) and the modified 3D model (4.3). In the following we consider the modified 3D model for different limits of the parameters $B$ and $A$.

5.1. Small and Large B Limit. We investigate the Fokker-Planck equation associated to (4.3)

$$
\begin{aligned}
\partial_{t} P= & -\boldsymbol{\tau} \cdot \nabla_{\xi} P+\frac{1}{\sin \theta} \frac{1}{B+1} \nabla V(\boldsymbol{\xi}) \cdot \partial_{\alpha}\left(\boldsymbol{n}_{1} P\right)+\frac{B}{B+1} \nabla V(\boldsymbol{\xi}) \cdot \partial_{\theta}\left(\boldsymbol{n}_{2} P\right) \\
& -\frac{1}{2} A^{2} \partial_{\theta}(\cot \theta P)+\frac{1}{2} \frac{A^{2}}{\sin ^{2} \theta} \partial_{\alpha \alpha} P+\frac{1}{2} B A^{2} \partial_{\theta \theta} P .
\end{aligned}
$$

The stationary density is given by

$$
P_{\text {stat }}(\boldsymbol{\xi}, \theta)=C \exp (-V(\boldsymbol{\xi}))(\sin \theta)^{\frac{1}{B}} .
$$

We note that in the case $B=1$ this is the Fokker-Planck equation and the stationary solution corresponding to the isotropic model (3.6). Next, we turn to the case $B$ tending to 0 . For $B=0$ the system of stochastic differential equations (4.3) decouples 
into a closed system of ordinary differential equations in $\left(\boldsymbol{\xi}_{3}, \theta\right)$

$$
\begin{aligned}
d \boldsymbol{\xi}_{3 t} & =\cos \theta_{t} d t \\
d \theta_{t} & =\frac{1}{2} A^{2} \cot \theta_{t} d t
\end{aligned}
$$

and a remaining system similar to the $2 \mathrm{D}$ model (2.1),

$$
\begin{aligned}
d \boldsymbol{\xi}_{1 t} & =\cos \alpha_{t} \sin \theta_{t} d t \\
d \boldsymbol{\xi}_{2 t} & =\sin \alpha_{t} \sin \theta_{t} d t \\
\sin \theta_{t} d \alpha_{t} & =-\nabla V\left(\boldsymbol{\xi}_{t}\right) \cdot \boldsymbol{n}_{1}\left(\alpha_{t}\right) d t+A d W_{t}^{(1)} .
\end{aligned}
$$

The solution of (5.3) with initial values $\boldsymbol{\xi}_{3}(0)=\boldsymbol{\xi}_{3_{0}}$ and $\theta(0)=\theta_{0}$ is

$$
\begin{aligned}
\theta(t) & =\arccos \left(\exp \left(-\frac{1}{2} A^{2} t\right) \cos \theta_{0}\right) \\
\boldsymbol{\xi}_{3}(t) & =\boldsymbol{\xi}_{3_{0}}+\frac{2}{A^{2}} \cos \theta_{0}-\frac{2}{A^{2}} \exp \left(-\frac{1}{2} A^{2} t\right) \cos \theta_{0} .
\end{aligned}
$$

In the large time limit $t \rightarrow \infty$ one obtains $\left(\xi_{3}, \theta\right)=\left(\boldsymbol{\xi}_{3_{0}}+\frac{2}{A^{2}} \cos \theta_{0}, \frac{\pi}{2}\right)$. Plugging this into the remaining system (5.4) we recover the $2 \mathrm{D}$ model (2.1). This means that after a transition time or if the initial values are suitably chosen, the 2-D model is recovered. Moreover, one directly observes that the stationary solution of the modified model (5.2) tends towards the stationary solution of the $2 \mathrm{D}$ model (2.6) if $B$ tends to zero:

$$
C(\sin \theta)^{\frac{1}{B}} \stackrel{B \rightarrow 0}{\longrightarrow} \delta_{\pi / 2} .
$$

5.2. Large Diffusion Limit and Reduced Model. In this section we investigate the large turbulence case with the limit $A \rightarrow \infty$. We start from the Fokker-Planck equation (5.1) associated to the modified model and scale the equation using $t^{\prime}=\varepsilon t$ and $A^{\prime}=\sqrt{\varepsilon} A$. This yields

$$
\begin{aligned}
\varepsilon \partial_{t} P^{\varepsilon}= & -\boldsymbol{\tau} \cdot \nabla_{\xi} P^{\varepsilon}+\frac{1}{\sin \theta} \frac{1}{B+1} \nabla V(\boldsymbol{\xi}) \cdot \partial_{\alpha}\left(\boldsymbol{n}_{1} P^{\varepsilon}\right)+\frac{B}{B+1} \nabla V(\boldsymbol{\xi}) \cdot \partial_{\theta}\left(\boldsymbol{n}_{2} P^{\varepsilon}\right) \\
& -\frac{1}{2} \frac{A^{2}}{\varepsilon} \partial_{\theta}\left(\cot \theta P^{\varepsilon}\right)+\frac{1}{2} \frac{A^{2}}{\varepsilon \sin ^{2} \theta} \partial_{\alpha \alpha} P^{\varepsilon}+\frac{1}{2} B \frac{A^{2}}{\varepsilon} \partial_{\theta \theta} P^{\varepsilon} .
\end{aligned}
$$

Plugging in the ansatz $P^{\varepsilon}=P^{0}+\varepsilon P^{1}+\ldots$ the leading order problem is

$$
\frac{1}{2} A^{2}\left(-\partial_{\theta}\left(\cot \theta P^{0}\right)+\frac{1}{\sin ^{2} \theta} \partial_{\alpha \alpha} P^{0}+B \partial_{\theta \theta} P^{0}\right)=0
$$

with solution

$$
P^{0}(\boldsymbol{\xi}, \theta, t)=C(\sin \theta)^{\frac{1}{B}} m^{0}(\boldsymbol{\xi}, t),
$$

where $C$ is a normalization constant depending on the parameter $B$. To next order we have

$$
\begin{aligned}
0= & -\boldsymbol{\tau} \cdot \nabla_{\xi} P^{0}+\frac{1}{\sin \theta} \frac{1}{B+1} \nabla V(\boldsymbol{\xi}) \cdot \partial_{\alpha}\left(\boldsymbol{n}_{1} P^{0}\right)+\frac{B}{B+1} \nabla V(\boldsymbol{\xi}) \cdot \partial_{\theta}\left(\boldsymbol{n}_{2} P^{0}\right) \\
& +\frac{1}{2} A^{2}\left(-\partial_{\theta}\left(\cot \theta P^{1}\right)+\frac{1}{\sin ^{2} \theta} \partial_{\alpha \alpha} P^{1}+B \partial_{\theta \theta} P^{1}\right) .
\end{aligned}
$$


Inserting $P^{0}$ yields

$$
-\partial_{\theta}\left(\cot \theta P^{1}\right)+\frac{1}{\sin ^{2} \theta} \partial_{\alpha \alpha} P^{1}+B \partial_{\theta \theta} P^{1}=C \frac{2}{A^{2}}\left(\nabla_{\xi} m^{0}+\nabla V(\boldsymbol{\xi}) m^{0}\right) \cdot \boldsymbol{\tau}(\sin \theta)^{\frac{1}{B}} .
$$

The solution of this equation is given by

$$
P^{1}(\boldsymbol{\xi}, \theta, \alpha, t)=-\frac{1}{B+1} \frac{2}{A^{2}} C(\sin \theta)^{\frac{1}{B}} \boldsymbol{\tau} \cdot\left(\nabla_{\xi} m^{0}+\nabla V(\boldsymbol{\xi}) m^{0}\right) .
$$

In a next step we integrate the scaled Fokker-Planck equation over the angles $\alpha$ and $\theta$. Defining $m^{\varepsilon}:=\int_{0}^{2 \pi} \int_{0}^{\pi} P^{\varepsilon} d \theta d \alpha$ we obtain

$$
\varepsilon \partial_{t} m^{\varepsilon}+\int_{0}^{2 \pi} \int_{0}^{\pi} \tau \cdot \nabla_{\xi} P^{\varepsilon} d \theta d \alpha=0
$$

To first order this is

$$
\partial_{t} m^{0}+\int_{0}^{2 \pi} \int_{0}^{\pi} \boldsymbol{\tau} \cdot \nabla_{\xi} P^{1} d \theta d \alpha=\mathcal{O}(\varepsilon)
$$

Applying the divergence relation $\nabla_{\xi} \cdot\left(\boldsymbol{\tau} P^{1}\right)=\boldsymbol{\tau} \cdot \nabla_{\xi} P^{1}+P^{1} \nabla_{\xi} \cdot \boldsymbol{\tau}=\boldsymbol{\tau} \cdot \nabla_{\xi} P^{1}$ we have

$$
\partial_{t} m^{0}+\nabla_{\xi} \cdot \int_{0}^{2 \pi} \int_{0}^{\pi} \tau P^{1} d \theta d \alpha=\mathcal{O}(\varepsilon)
$$

and after replacing $P^{1}$ we get

$$
\partial_{t} m^{0}-\nabla_{\xi} \cdot \int_{0}^{2 \pi} \int_{0}^{\pi} \boldsymbol{\tau} \frac{1}{B+1} \frac{2}{A^{2}} C(\sin \theta)^{\frac{1}{B}} \boldsymbol{\tau} \cdot\left(\nabla_{\xi} m^{0}+\nabla V(\boldsymbol{\xi}) m^{0}\right) d \theta d \alpha=\mathcal{O}(\varepsilon) .
$$

After evaluating the integral and taking the limit $\varepsilon \rightarrow 0$ we obtain the reduced model

$$
\partial_{t} m^{0}-\frac{1}{A^{2}(1+2 B)} \nabla_{\xi} \cdot D\left(\nabla_{\xi} m^{0}+\nabla V(\boldsymbol{\xi}) m^{0}\right)=0
$$

with

$$
D=\operatorname{diag}\left\{1,1, \frac{2 B}{(1+B)}\right\}
$$

or the system of stochastic differential equations

$$
d \boldsymbol{\xi}_{t}=-\frac{1}{1+2 B} \frac{1}{A^{2}} D \nabla V\left(\boldsymbol{\xi}_{t}\right) d t+\sqrt{\frac{2}{1+2 B}} \frac{1}{A} \sqrt{D} d \boldsymbol{W}_{t}
$$

We note, that for $B=0$ we recover the reduced $2 \mathrm{D}$ model $(2.7)$.

REMARK 6. The limiting process for $A \rightarrow \infty$ in the case of a moving conveyor belt is given by

$$
d \boldsymbol{\xi}_{t}=-\left(\frac{1}{1+2 B} \frac{1}{A^{2}} D \nabla V\left(\boldsymbol{\xi}_{t}\right)-\kappa \boldsymbol{e}_{1}\right) d t+\sqrt{\frac{2}{1+2 B}} \frac{1}{A} \sqrt{D} d \boldsymbol{W}_{t}
$$



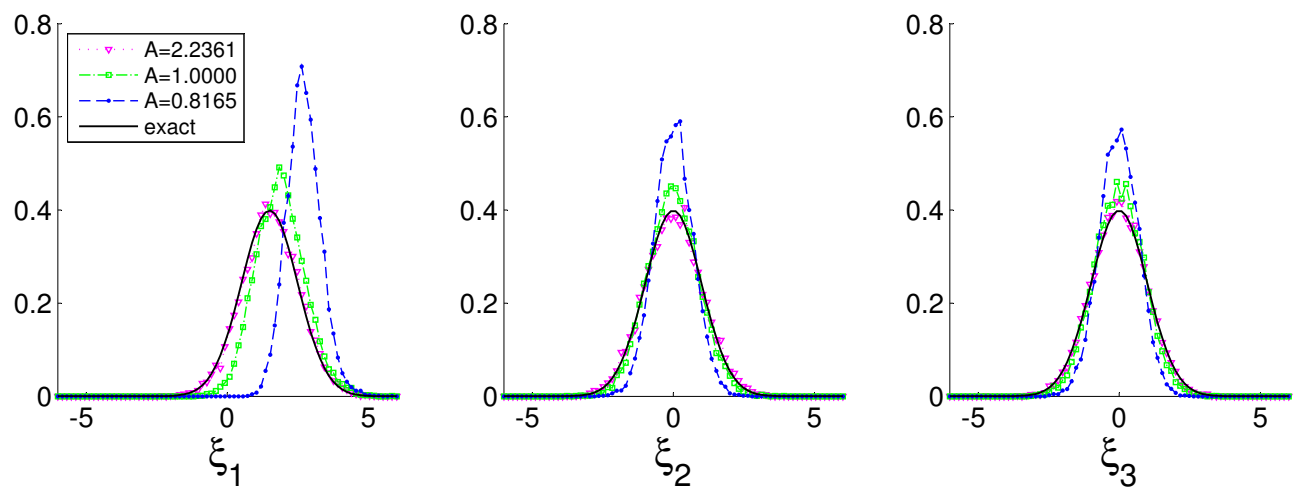

Fig. 5.1: Stationary marginal densities of $\boldsymbol{\xi}$-components for different values of $A$ and fixed $q=0.5$

compare [3] for the two-dimensional case.

To investigate the practical relevance of the reduced model numerically we compare the isotropic 3D model process with moving belt (3.5) with the reduced model (5.6) with $B=1$. The potential is chosen as $V(\boldsymbol{\xi})=|\boldsymbol{\xi}|^{2} / 2$. The stationary density of (5.6) reads

$$
P_{\text {stat }}(\boldsymbol{\xi})=C \exp \left(-\frac{\left(\xi_{1}-(2 B+1) \kappa A^{2}\right)^{2}}{2}-\frac{\xi_{2}^{2}}{2}-\frac{\xi_{3}^{2}}{2}\right)
$$

which is independent of the scaling for $\kappa A^{2}=q, q \in \mathbb{R}$. Figure 5.1 shows the stationary marginal densities for the components $\xi_{1}, \xi_{2}$ and $\xi_{3}$ for (3.5) with different values of $A$ and fixed $q=0.5$ and the above stationary density of (5.6) for $B=1$. Whereas the marginal densities differ for $A<1$, the approximation is qualitatively better for increasing $A$. Good agreement can be observed for $A>2$. Similar results can be shown for the modified 3D model $(B<1)$. Thus the reduced models might be seen as alternatives to the original models even for moderate values of $A$. However, we note that in the limit we loose regularity of the fiber path. Moreover, the inextensibility of the fiber is not anymore preserved.

REMARK 7. In [6] also the small diffusion limit $A \rightarrow 0$ has been considered for the $2 D$ case using a representation of the equations as a stochastic Hamiltonian system and the method of stochastic averaging, see also [1, 2]. Similarly, the 3D system can be rewritten in Hamiltonian form. However, in contrast to the $2 D$ case where the reduced Hamiltonian system has been a 2-dimensional system, it is in the present case a 4-dimensional equation allowing for 3 invariants, which are not as straightforwardly determined.

5.3. The Large Coiling Force Limit. In this section we investigate a hyperbolic scaling, the large coiling force and large diffusion limit with $A^{\prime}=A / \sqrt{\epsilon}$ and 
$V^{\prime}=V / \epsilon$. We start from the scaled Fokker-Planck equation

$$
\begin{aligned}
\partial_{t} P^{\varepsilon}= & -\boldsymbol{\tau} \cdot \nabla_{\xi} P^{\varepsilon}+\frac{1}{\varepsilon} \frac{1}{\sin \theta} \frac{1}{B+1} \nabla V(\boldsymbol{\xi}) \cdot \partial_{\alpha}\left(\boldsymbol{n}_{1} P^{\varepsilon}\right)+\frac{1}{\varepsilon} \frac{B}{B+1} \nabla V(\boldsymbol{\xi}) \cdot \partial_{\theta}\left(\boldsymbol{n}_{2} P^{\varepsilon}\right) \\
& -\frac{1}{2} \frac{A^{2}}{\varepsilon} \partial_{\theta}\left(\cot \theta P^{\varepsilon}\right)+\frac{1}{2} \frac{A^{2}}{\varepsilon \sin ^{2} \theta} \partial_{\alpha \alpha} P^{\varepsilon}+\frac{1}{2} B \frac{A^{2}}{\varepsilon} \partial_{\theta \theta} P^{\varepsilon} .
\end{aligned}
$$

To zeroth order this is

$$
\begin{array}{r}
\frac{1}{\sin \theta} \frac{1}{B+1} \nabla V(\boldsymbol{\xi}) \cdot \partial_{\alpha}\left(\boldsymbol{n}_{1} P^{0}\right)+\frac{B}{B+1} \nabla V(\boldsymbol{\xi}) \cdot \partial_{\theta}\left(\boldsymbol{n}_{2} P^{0}\right) \\
-\frac{1}{2} A^{2} \partial_{\theta}\left(\cot \theta P^{0}\right)+\frac{1}{2} \frac{A^{2}}{\sin ^{2} \theta} \partial_{\alpha \alpha} P^{0}+\frac{1}{2} B A^{2} \partial_{\theta \theta} P^{0}=0
\end{array}
$$

with the solution

$$
P^{0}(\boldsymbol{\xi}, \alpha, \theta, t)=\frac{\rho(\boldsymbol{\xi}, t)}{N(\boldsymbol{\xi})}(\sin \theta)^{\frac{1}{B}} \exp \left(-\frac{1}{A^{2}} \frac{2}{B+1} \boldsymbol{\tau} \cdot \nabla V\right)
$$

with

$$
N(\boldsymbol{\xi})=\int(\sin \theta)^{\frac{1}{B}} \exp \left(-\frac{1}{A^{2}} \frac{2}{B+1} \boldsymbol{\tau} \cdot \nabla V\right) d \alpha d \theta .
$$

Integrating the scaled Fokker-Planck equation over $\alpha$ and $\theta$ yields up to order $\epsilon$

$$
\partial_{t} \rho+\nabla_{\xi} \cdot \int \tau P^{0} d \alpha d \theta=0
$$

This can be rewritten as

$$
\partial_{t} \rho+\nabla_{\xi} \cdot(\rho \boldsymbol{U})=0
$$

with

$$
\boldsymbol{U}=\boldsymbol{U}(\boldsymbol{\xi})=\frac{1}{N(\boldsymbol{\xi})} \int \boldsymbol{\tau}(\sin \theta)^{\frac{1}{B}} \exp \left(-\frac{1}{A^{2}} \frac{2}{B+1} \boldsymbol{\tau} \cdot \nabla V\right) d \theta d \alpha .
$$

Under suitable assumptions concerning the symmetry of the process, for example, in the isotropic case $B=1$ and assuming that $V(\boldsymbol{\xi})=V(|\boldsymbol{\xi}|)$ we have $N(\boldsymbol{\xi})=N(|\boldsymbol{\xi}|)$ and we can rewrite $\boldsymbol{U}$ as

$$
\boldsymbol{U}(\boldsymbol{\xi})=\lambda(|\boldsymbol{\xi}|) \boldsymbol{\xi}
$$

with

$$
\lambda(|\boldsymbol{\xi}|)=\frac{1}{V^{\prime}(|\boldsymbol{\xi}|)|\boldsymbol{\xi}| N(|\boldsymbol{\xi}|)} \int \nabla V \cdot \boldsymbol{\tau} \sin \theta \exp \left(-\frac{1}{A^{2}} \boldsymbol{\tau} \cdot \nabla V\right) d \theta d \alpha .
$$

Thus, the limit equation is in such a case given by

$$
\partial_{t} \rho+\nabla_{\xi} \cdot(\rho \lambda(|\boldsymbol{\xi}|) \boldsymbol{\xi})=0 .
$$

In this case one shows that $\lambda$ is negative, i.e. the vector field $\boldsymbol{U}$ points towards the origin. For $B=1$ and the potential $V(\boldsymbol{\xi})=|\boldsymbol{\xi}|^{2} / 2$ we plot $|\boldsymbol{\xi}| \lambda(|\boldsymbol{\xi}|)$ in Figure 5.2. For general $B<1$, one can show that the vector field $\boldsymbol{U}$ is symmetric with respect to the $\boldsymbol{e}_{3}$-axis, compare Figure 5.2.

REMARK 8. An equivalent result can be proven with the same method in the 2D case. 

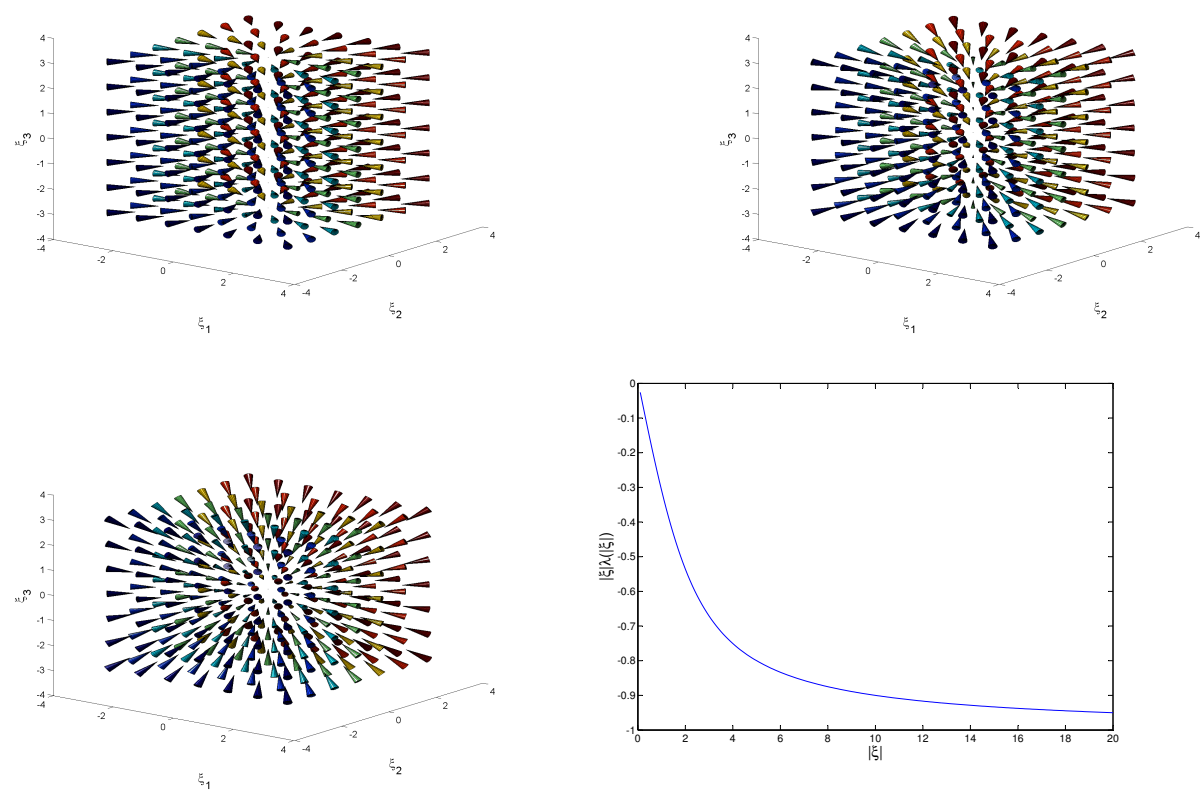

Fig. 5.2: Plots of the vector field $\boldsymbol{U}$ for different parameter $B=0 ; 0.1 ; 1$ and plot of $|\boldsymbol{\xi}| \lambda(|\boldsymbol{\xi}|)$ on the bottom right for the isotropic case $(B=1)$ and fixed $A$

5.4. Relations between the Models. Figure 5.3 shows the scaling limits of the modified 3D model (4.3).

6. Numerical Simulations and Identification of the Parameters. In the 2D case the parameter $A$ and the shape of the potential $V$ have been estimated in [10] for different production processes. There, full simulations of a single representative fiber have been performed with the software tool FIDYST ${ }^{1}$, see $[12,8]$ for a description of the algorithms. The noise amplitude $A$ and the potential $V$ in the $2 \mathrm{D}$ model (2.4) have been identified on the basis of the full simulation. Figure 6.1 shows as an example the fiber trajectories computed by the calibrated 2D model and, in comparison, an underlying full FIDYST simulation. The fiber mass distribution is captured qualitatively well in the surrogate model. The potential in $\xi_{3}$ might be assumed as confining potential which models the impenetrable conveyor belt.

It remains to give a method to determine the distribution of the $\theta$-angle in a real material and translate this into the corresponding parameter $B$. Therefore we assume that the measurement of the $\theta$-angle in a real nonwoven leads to an axisymmetric distribution in $\theta$ with mean $m_{\theta}=\pi / 2$ and variance $\operatorname{var}_{\theta}=\sigma_{\theta}^{2}$. Then the parameter $B$ in the stationary $\theta$-distribution $P_{B}(\theta)=C \sin \theta^{\frac{1}{B}}$ is chosen such that the variance of this distribution coincides with $\operatorname{var}_{\theta}$.

In Figure 6.2 an example of a virtual fleece generated by the modified 3D model is shown, where we simulate 10 fibers on a moving conveyor belt with assumed speed ratio $\kappa=0.0238$. The distance between two spinning nozzles is chosen as $d=2.5$.

\footnotetext{
${ }^{1}$ FIDYST:Fiber Dynamics Simulation Tool developed at Fraunhofer ITWM, Kaiserslautern
} 


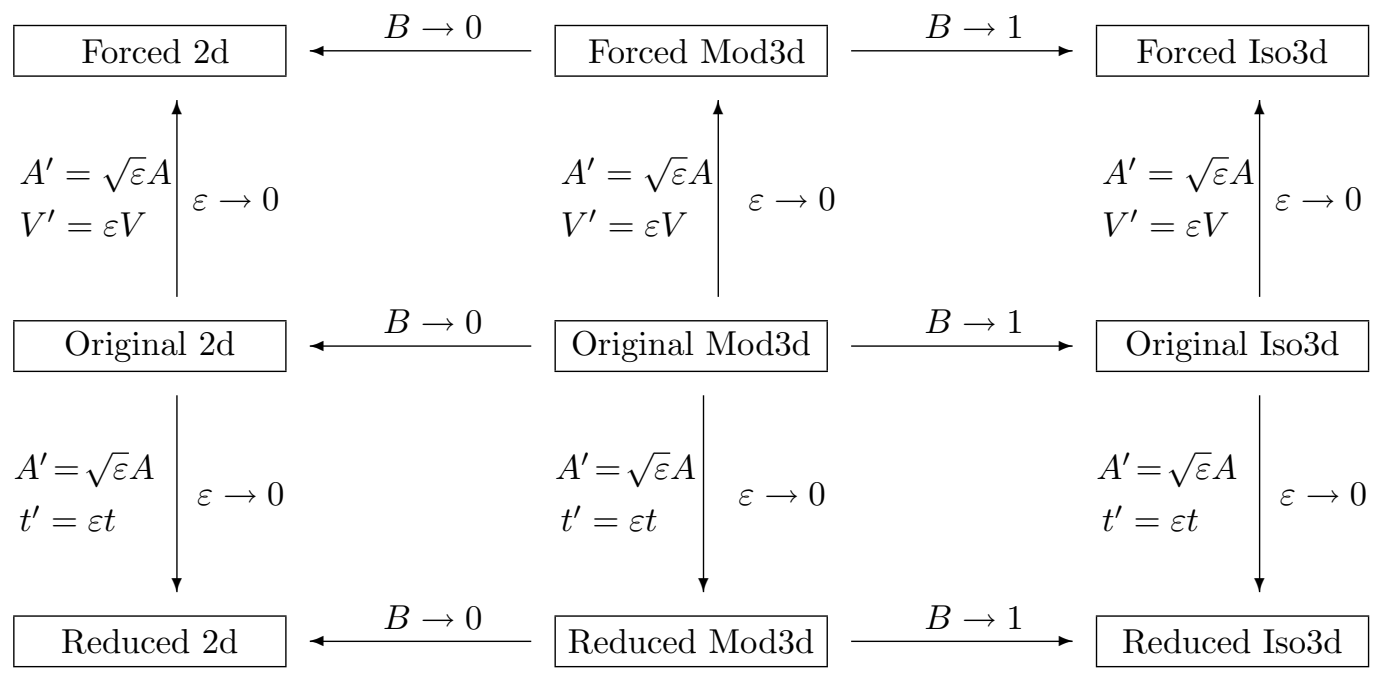

Fig. 5.3: Asymptotic limits of the 3D fiber model for different scalings.
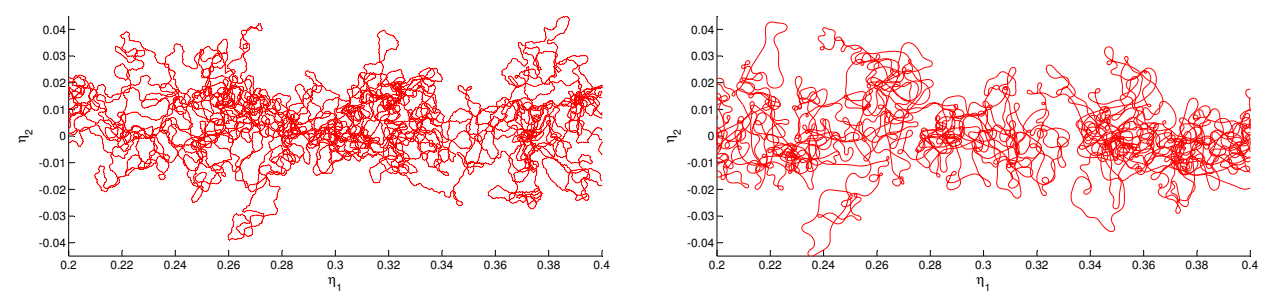

Fig. 6.1: Comparison of fiber path associated to the adapted 2D surrogate model (left) and the FIDYST simulation (right)

$10^{-3}$ and the variance of the $\theta$-distribution as $\sigma_{\theta}^{2}=0.0169$ which corresponds to a parameter $B=0.0171$. The throwing ranges $\sigma_{1}, \sigma_{2}$ in the standard buckling potential $V(\boldsymbol{\xi})=\left(\xi_{1}^{2} / \sigma_{1}^{2}+\xi_{2}^{2} / \sigma_{2}^{2}\right) / 2$ and the noise amplitude $A$ are determined by the parameter identification from FIDYST data. The throwing range in $z$-direction or the fleece thickness is supposed to be $d_{\text {fleece }}=0.01$.

7. Conclusion and Outlook . In this paper we derived a new 3D model for the fiber lay down process in technical textile production taking into account the anisotropic orientation of the fibers in the resulting fleece. In future work we plan to apply the model to real fiber process problems, where the distribution of the angle $\theta$ can be obtained by a computer tomography of the non-woven and associated image analysis. Moreover, from the theoretical point of view the convergence to equilibrium of the 3D models will be discussed with methods developed in [4]. See [5] for the 2-D 

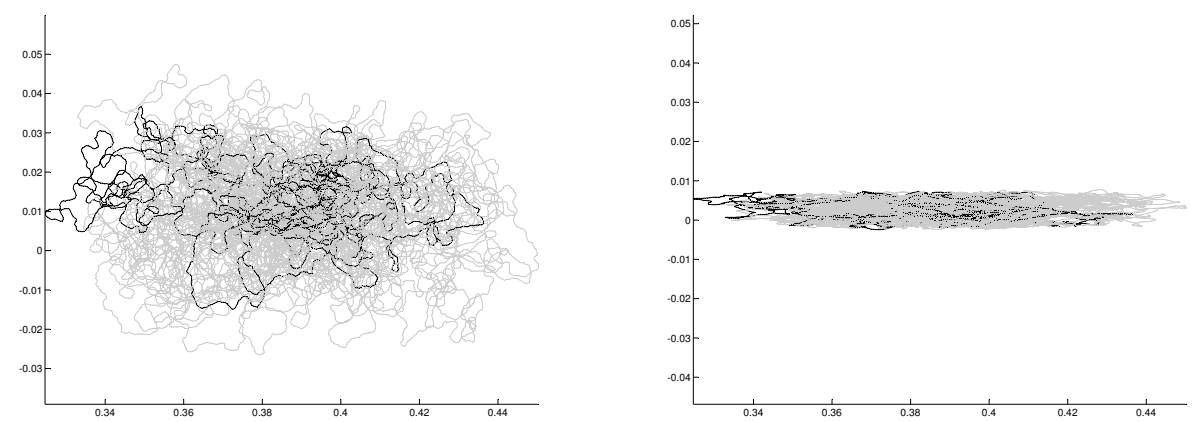

Fig. 6.2: Example of a virtual fleece (10 fibers) with top view (left) and side view (right). A representative single fiber is emphasized as darker curve.

case. The drawback of non-differentiable fiber path can be overcome by replacing the Wiener Process by an Ornstein-Uhlenback process leading to a more realistic smooth model [11] analogous to the 2-D case, see [8].

Acknowledgments. This work has been supported by Deutsche Forschungsgemeinschaft (DFG), WE 2003/3-1, KL 1105/18-1 and by Bundesministerium für Bildung und Forschung (BMBF), Verbundprojekt ProFil, 03MS606

\section{REFERENCES}

[1] S. Albeverio and A. Klar, Longtime behaviour of nonlinear stochastic oscillators, J. Math. Phys. 35(8) 4005-4027, 1994.

[2] S. Albeverio and A. Klar, Longtime behaviour of stochastic Hamiltonian systems, Potential Analysis 12, 281-297, 2000.

[3] L. Bonilla and T. Götz and A. Klar and N. Marheineke and R. Wegener, Hydrodynamic limit of a Fokker-Planck equation describing fiber lay-down processes, SIAM J. Appl. Math. 68(3), 648-665, 2007

[4] J. Dolbeault and C. Mouhot and C. Schmeiser Hypocoercivity for linear equations conserving mass, preprint

[5] J. Dolbeault and A. Klar and C. Mouhot and C. Schmeiser Hypocoercivity and a Fokker-Planck equation for fiber lay-down, preprint

[6] T. Götz and A. Klar and N. Marheineke and R. Wegener, A stochastic model and associated Fokker-Planck equation for the fiber lay-down process in nonwoven production processes, SIAM J. Appl. Math. 67(6), 1704-1717, 2007

[7] M. Grothaus and A. Klar, Ergodicity and rate of convergence for a non-sectorial fiber lay-down process, SIAM J. Math. Anal. 40(3), 968-983, 2008

[8] M. Herty and A. Klar and S. Motsch and F. Olawsky, A smooth model for fibre lay-down processes and its diffusion approximation, KRM 2 (3), 480-502, 2009

[9] A. Klar and P. Reuterswärd and M. Seaïd A semi-Lagrangian method for a Fokker-Planck equation describing fiber dynamics, J.Sci.Comp. 38(3), 349-367, 2009

[10] A. Klar and N. Marheineke, and R. Wegener, Hierarchy of mathematical models for production processes of technical textiles, ZAMM 89(12), 941-961, 2009

[11] A. Klar and J. Maringer, and R. Wegener A Smooth 3D Model for Fiber Lay-down in Nonwoven Production Processes, preprint

[12] N. Marheineke and R. Wegener, Fiber dynamics in turbulent flows: General modeling framework, SIAM J. Appl. Math. 66(5), 1703-1726, 2006

[13] N. Marheineke, and R. Wegener, Fiber dynamics in turbulent flows: Specific Taylor drag, SIAM J. Appl. Math. 68(1), 1-23, 2007

[14] Bernt $\varnothing$ ksendal, Stochastic differential equations, Springer, 2007 
[15] D.W. Stroock, On the growth of stochastic integrals, Z.Wahr. verw. Geb. 18, 340-344 


\section{Published reports of the Fraunhofer ITWM}

The PDF-files of the following reports are available under:

\section{www.itwm.fraunhofer.de/de/ zentral_berichte/berichte}

1. D. Hietel, K. Steiner, J. Struckmeier A Finite - Volume Particle Method for Compressible Flows (19 pages, 1998)

2. M. Feldmann, S. Seibold

Damage Diagnosis of Rotors: Application of Hilbert Transform and Multi-Hypothesis Testing

Keywords: Hilbert transform, damage diagnosis,

Kalman filtering, non-linear dynamics

(23 pages, 1998)

3. Y. Ben-Haim, S. Seibold

Robust Reliability of Diagnostic MultiHypothesis Algorithms: Application to Rotating Machinery

Keywords: Robust reliability, convex models, Kalman filtering, multi-hypothesis diagnosis, rotating machinery, crack diagnosis

(24 pages, 1998)

\section{F.-Th. Lentes, N. Siedow}

Three-dimensional Radiative Heat Transfer in Glass Cooling Processes

(23 pages, 1998)

5. A. Klar, R. Wegener

A hierarchy of models for multilane vehicular traffic

Part I: Modeling

(23 pages, 1998)

Part II: Numerical and stochastic investigations (17 pages, 1998)

\section{A. Klar, N. Siedow}

Boundary Layers and Domain Decomposition for Radiative Heat Transfer and Diffusion Equations: Applications to Glass Manufacturing Processes

(24 pages, 1998)

7. I. Choquet

Heterogeneous catalysis modelling and numerical simulation in rarified gas flows Part l: Coverage locally at equilibrium (24 pages, 1998)

8. J. Ohser, B. Steinbach, C. Lang Efficient Texture Analysis of Binary Images (17 pages, 1998)

9. J. Orlik

Homogenization for viscoelasticity of the integral type with aging and shrinkage (20 pages, 1998)

10. J. Mohring

Helmholtz Resonators with Large Aperture (21 pages, 1998)
11. H. W. Hamacher, A. Schöbel On Center Cycles in Grid Graphs (15 pages, 1998)

12. H. W. Hamacher, K.-H. Küfer Inverse radiation therapy planning a multiple objective optimisation approach (14 pages, 1999)

13. C. Lang, J. Ohser, R. Hilfer On the Analysis of Spatial Binary Images (20 pages, 1999)

14. M. Junk

On the Construction of Discrete Equilibrium Distributions for Kinetic Schemes (24 pages, 1999)

15. M. Junk, S. V. Raghurame Rao

A new discrete velocity method for NavierStokes equations

(20 pages, 1999)

16. H. Neunzert

Mathematics as a Key to Key Technologies (39 pages, 1999)

17. J. Ohser, K. Sandau

Considerations about the Estimation of the Size Distribution in Wicksell's Corpuscle Problem

(18 pages, 1999)

18. E. Carrizosa, H. W. Hamacher, R. Klein, S. Nickel

Solving nonconvex planar location problems by finite dominating sets

Keywords: Continuous Location, Polyhedral Gauges, Finite Dominating Sets, Approximation, Sandwich Algorithm, Greedy Algorithm

(19 pages, 2000)

\section{A. Becker}

A Review on Image Distortion Measures Keywords: Distortion measure, human visual system (26 pages, 2000)

20. H. W. Hamacher, M. Labbé, S. Nickel, T. Sonneborn

Polyhedral Properties of the Uncapacitated Multiple Allocation Hub Location Problem Keywords: integer programming, hub location, facility location, valid inequalities, facets, branch and cut (21 pages, 2000)

21. H. W. Hamacher, A. Schöbel

Design of Zone Tariff Systems in Public Transportation

(30 pages, 2001)

22. D. Hietel, M. Junk, R. Keck, D. Teleaga The Finite-Volume-Particle Method for Conservation Laws (16 pages, 2001)

23. T. Bender, H. Hennes, J. Kalcsics, M. T. Melo, S. Nickel

Location Software and Interface with GIS and Supply Chain Management Keywords: facility location, software development, geographical information systems, supply chain management

(48 pages, 2001)
24. H. W. Hamacher, S. A. Tjandra Mathematical Modelling of Evacuation Problems: A State of Art (44 pages, 2001)

25. J. Kuhnert, S. Tiwari

Grid free method for solving the Poisson equation

Keywords: Poisson equation, Least squares method, Grid free method

(19 pages, 2001)

26. T. Götz, H. Rave, D. Reinel-Bitzer, K. Steiner, $\mathrm{H}$. Tiemeier

Simulation of the fiber spinning process Keywords: Melt spinning, fiber model, Lattice Boltzmann, CFD

(19 pages, 2001)

\section{A. Zemitis}

On interaction of a liquid film with an obstacle Keywords: impinging jets, liquid film, models, numerical solution, shape

22 pages, 2001)

\section{I. Ginzburg, K. Steiner}

Free surface lattice-Boltzmann method to model the filling of expanding cavities by Bingham Fluids

Keywords: Generalized LBE, free-surface phenomena interface boundary conditions, filling processes, Bing ham viscoplastic model, regularized models

(22 pages, 2001)

\section{H. Neunzert}

"Denn nichts ist für den Menschen als Menschen etwas wert, was er nicht mit Leidenschaft tun kann

Vortrag anlässlich der Verleihung des Akademiepreises des Landes RheinlandPfalz am 21.11.2001

Keywords: Lehre, Forschung, angewandte Mathematik, Mehrskalenanalyse, Strömungsmechanik (18 pages, 2001)

30. J. Kuhnert, S. Tiwari

Finite pointset method based on the projection method for simulations of the incompressible Navier-Stokes equations

Keywords: Incompressible Navier-Stokes equations, Meshfree method, Projection method, Particle scheme, Least squares approximation

AMS subject classification: 76D05, 76M28

(25 pages, 2001)

31. R. Korn, M. Krekel

Optimal Portfolios with Fixed Consumption or Income Streams

Keywords: Portfolio optimisation, stochastic control, HJB equation, discretisation of control problems (23 pages, 2002)

32. M. Krekel

Optimal portfolios with a loan dependent credit spread

Keywords: Portfolio optimisation, stochastic control, HJB equation, credit spread, log utility, power utility, non-linear wealth dynamics

(25 pages, 2002)

33. J. Ohser, W. Nagel, K. Schladitz

The Euler number of discretized sets - on the choice of adjacency in homogeneous lattices Keywords: image analysis, Euler number, neighborhod relationships, cuboidal lattice

(32 pages, 2002) 
34. I. Ginzburg, K. Steiner

Lattice Boltzmann Model for Free-Surface flow and Its Application to Filling Process in Casting

Keywords: Lattice Boltzmann models; free-surface phenomena; interface boundary conditions; filling processes; injection molding; volume of fluid method; interface boundary conditions; advection-schemes; upwind-schemes

(54 pages, 2002)

35. M. Günther, A. Klar, T. Materne, R. Wegener

Multivalued fundamental diagrams and stop and go waves for continuum traffic equations Keywords: traffic flow, macroscopic equations, kinetic derivation, multivalued fundamental diagram, stop and go waves, phase transitions

(25 pages, 2002)

36. S. Feldmann, P. Lang, D. Prätzel-Wolters Parameter influence on the zeros of network determinants

Keywords: Networks, Equicofactor matrix polynomials, Realization theory, Matrix perturbation theory

(30 pages, 2002)

\section{K. Koch, J. Ohser, K. Schladitz} Spectral theory for random closed sets and estimating the covariance via frequency space

Keywords: Random set, Bartlett spectrum, fast Fourier transform, power spectrum

(28 pages, 2002)

\section{D. d'Humières, I. Ginzburg}

Multi-reflection boundary conditions for lattice Boltzmann models

Keywords: lattice Boltzmann equation, boudary condistions, bounce-back rule, Navier-Stokes equation

(72 pages, 2002)

\section{R. Korn}

\section{Elementare Finanzmathematik}

Keywords: Finanzmathematik, Aktien, Optionen, Portfolio-Optimierung, Börse, Lehrerweiterbildung, Mathematikunterricht

(98 pages, 2002)

40. J. Kallrath, M. C. Müller, S. Nickel

Batch Presorting Problems:

Models and Complexity Results

Keywords: Complexity theory, Integer programming,

Assigment, Logistics

(19 pages, 2002)

\section{J. Linn}

On the frame-invariant description of the phase space of the Folgar-Tucker equation Key words: fiber orientation, Folgar-Tucker equation, injection molding

(5 pages, 2003)

\section{T. Hanne, S. Nickel}

A Multi-Objective Evolutionary Algorithm for Scheduling and Inspection Planning in Software Development Projects

Key words: multiple objective programming, project management and scheduling, software development, evolutionary algorithms, efficient set

(29 pages, 2003)

43. T. Bortfeld, K.-H. Küfer, M. Monz,

A. Scherrer, C. Thieke, H. Trinkaus

Intensity-Modulated Radiotherapy - A Large Scale Multi-Criteria Programming Problem
Keywords: multiple criteria optimization, representative systems of Pareto solutions, adaptive triangulation, clustering and disaggregation techniques, visualization of Pareto solutions, medical physics, external beam radiotherapy planning, intensity modulated radiotherapy (31 pages, 2003)

\section{T. Halfmann, T. Wichmann}

Overview of Symbolic Methods in Industrial Analog Circuit Design

Keywords: $C A D$, automated analog circuit design, sym bolic analysis, computer algebra, behavioral modeling, system simulation, circuit sizing, macro modeling, differential-algebraic equations, index

(17 pages, 2003)

\section{S. E. Mikhailov, J. Orlik}

Asymptotic Homogenisation in Strength and Fatigue Durability Analysis of Composites

Keywords: multiscale structures, asymptotic homogenization, strength, fatigue, singularity, non-local conditions

(14 pages, 2003)

46. P. Domínguez-Marín, P. Hansen, N. Mladenovic, S. Nickel Heuristic Procedures for Solving the Discrete Ordered Median Problem Keywords: genetic algorithms, variable neighborhood search, discrete facility location

(31 pages, 2003)

47. N. Boland, P. Domínguez-Marín, S. Nickel, J. Puerto

Exact Procedures for Solving the Discrete Ordered Median Problem

Keywords: discrete location, Integer programming

(41 pages, 2003)

\section{S. Feldmann, P. Lang}

Padé-like reduction of stable discrete linear systems preserving their stability

Keywords: Discrete linear systems, model reduction,

stability, Hankel matrix, Stein equation

(16 pages, 2003)

49. J. Kallrath, S. Nickel

A Polynomial Case of the Batch Presorting

\section{Problem}

Keywords: batch presorting problem, online optimization, competetive analysis, polynomial algorithms, logistics (17 pages, 2003)

\section{T. Hanne, H. L. Trinkaus}

knowCube for MCDM -

Visual and Interactive Support for

Multicriteria Decision Making

Key words: Multicriteria decision making, knowledge management, decision support systems, visual interfaces, interactive navigation, real-life applications. (26 pages, 2003)

51. O. lliev, V. Laptev

On Numerical Simulation of Flow Through Oil Filters

Keywords: oil filters, coupled flow in plain and porous media, Navier-Stokes, Brinkman, numerical simulation (8 pages, 2003)

52. W. Dörfler, O. Iliev, D. Stoyanov, D. Vassileva On a Multigrid Adaptive Refinement Solver for Saturated Non-Newtonian Flow in Porous Media

Keywords: Nonlinear multigrid, adaptive refinement, non-Newtonian flow in porous media

(17 pages, 2003)
53. S. Kruse

On the Pricing of Forward Starting Options under Stochastic Volatility

Keywords: Option pricing, forward starting options, Heston model, stochastic volatility, cliquet options (11 pages, 2003)

54. O. Iliev, D. Stoyanov

Multigrid - adaptive local refinement solver for incompressible flows

Keywords: Navier-Stokes equations, incompressible flow projection-type splitting, SIMPLE, multigrid methods, adaptive local refinement, lid-driven flow in a cavity (37 pages, 2003)

\section{V. Starikovicius}

The multiphase flow and heat transfer in porous media

Keywords: Two-phase flow in porous media, various formulations, global pressure, multiphase mixture model, numerical simulation

(30 pages, 2003)

\section{P. Lang, A. Sarishvili, A. Wirsen}

Blocked neural networks for knowledge extraction in the software development process Keywords: Blocked Neural Networks, Nonlinear Regression, Knowledge Extraction, Code Inspection (21 pages, 2003)

\section{H. Knaf, P. Lang, S. Zeiser}

Diagnosis aiding in Regulation

Thermography using Fuzzy Logic

Keywords: fuzzy logic, knowledge representation, expert system

(22 pages, 2003)

58. M. T. Melo, S. Nickel, F. Saldanha da Gama Largescale models for dynamic multicommodity capacitated facility location Keywords: supply chain management, strategic planning, dynamic location, modeling (40 pages, 2003)

\section{J. Orlik}

Homogenization for contact problems with periodically rough surfaces

Keywords: asymptotic homogenization, contact problems (28 pages, 2004)

60. A. Scherrer, K.-H. Küfer, M. Monz,

F. Alonso, T. Bortfeld

IMRT planning on adaptive volume structures - a significant advance of computational complexity

Keywords: Intensity-modulated radiation therapy (IMRT), inverse treatment planning, adaptive volume structures, hierarchical clustering, local refinement, adaptive clustering, convex programming, mesh generation, multi-grid methods

(24 pages, 2004)

\section{D. Kehrwald}

Parallel lattice Boltzmann simulation of complex flows

Keywords: Lattice Boltzmann methods, parallel computing, microstructure simulation, virtual material design, pseudo-plastic fluids, liquid composite moulding (12 pages, 2004)

62. O. Iliev, J. Linn, M. Moog, D. Niedziela, V. Starikovicius

On the Performance of Certain Iterative Solvers for Coupled Systems Arising in Discretization of Non-Newtonian Flow Equations 
Keywords: Performance of iterative solvers, Preconditioners, Non-Newtonian flow

(17 pages, 2004)

\section{R. Ciegis, O. Iliev, S. Rief, K. Steiner}

On Modelling and Simulation of Different Regimes for Liquid Polymer Moulding Keywords: Liquid Polymer Moulding, Modelling, Simulation, Infiltration, Front Propagation, non-Newtonian flow in porous media

(43 pages, 2004)

\section{T. Hanne, H. Neu}

Simulating Human Resources in

\section{Software Development Processes}

Keywords: Human resource modeling, software process, productivity, human factors, learning curve

(14 pages, 2004)

\section{O. Iliev, A. Mikelic, P. Popov}

Fluid structure interaction problems in de-

formable porous media: Toward permeability of deformable porous media

Keywords: fluid-structure interaction, deformable porous media, upscaling, linear elasticity, stokes, finite elements

(28 pages, 2004)

66. F. Gaspar, O. Iliev, F. Lisbona, A. Naumovich, P. Vabishchevich

On numerical solution of 1-D poroelasticity equations in a multilayered domain Keywords: poroelasticity, multilayered material, finite volume discretization, MAC type grid

(41 pages, 2004)

67. J. Ohser, K. Schladitz, K. Koch, M. Nöthe Diffraction by image processing and its application in materials science

Keywords: porous microstructure, image analysis, random set, fast Fourier transform, power spectrum, Bartlett spectrum

(13 pages, 2004)

\section{H. Neunzert}

\section{Mathematics as a Technology: Challenges} for the next 10 Years

Keywords: applied mathematics, technology, modelling, simulation, visualization, optimization, glass processing, spinning processes, fiber-fluid interaction, trubulence effects, topological optimization, multicriteria optimiza tion, Uncertainty and Risk, financial mathematics, Malliavin calculus, Monte-Carlo methods, virtual material design, filtration, bio-informatics, system biology

(29 pages, 2004)

69. R. Ewing, O. lliev, R. Lazarov, A. Naumovich On convergence of certain finite difference discretizations for 1D poroelasticity interface problems

Keywords: poroelasticity, multilayered material, finite volume discretizations, MAC type grid, error estimates (26 pages, 2004 )

70. W. Dörfler, O. Iliev, D. Stoyanov, D. Vassileva On Efficient Simulation of Non-Newtonian Flow in Saturated Porous Media with a Multigrid Adaptive Refinement Solver Keywords: Nonlinear multigrid, adaptive renement non-Newtonian in porous media (25 pages, 2004)

\section{J. Kalcsics, S. Nickel, M. Schröde}

Towards a Unified Territory Design Approach - Applications, Algorithms and GIS Integration Keywords: territory desgin, political districting, sales territory alignment, optimization algorithms, Geographical Information Systems

(40 pages, 2005)
72. K. Schladitz, S Peters, D Reinel-Bitzer, A. Wiegmann, J. Ohser

Design of acoustic trim based on geometric modeling and flow simulation for non-woven Keywords: random system of fibers, Poisson line process, flow resistivity, acoustic absorption, Lattice-Boltzmann method, non-woven (21 pages, 2005)

\section{V. Rutka, A. Wiegmann}

Explicit Jump Immersed Interface Method for virtual material design of the effective elastic moduli of composite materials Keywords: virtual material design, explicit jump immersed interface method, effective elastic moduli, composite materials

(22 pages, 2005)

\section{T. Hanne}

Eine Übersicht zum Scheduling von Baustellen Keywords: Projektplanung, Scheduling, Bauplanung, Bauindustrie

(32 pages, 2005)

75. J. Linn

The Folgar-Tucker Model as a Differetial Algebraic System for Fiber Orientation Calculation

Keywords: fiber orientation, Folgar-Tucker model, invariants, algebraic constraints, phase space, trace stability

(15 pages, 2005)

76. M. Speckert, K. Dreßler, H. Mauch, A. Lion, G. J. Wierda

Simulation eines neuartigen Prüfsystems für Achserprobungen durch MKS-Modellierung einschließlich Regelung Keywords: virtual test rig, suspension testing, multibody simulation, modeling hexapod test rig, opti mization of test rig configuration

(20 pages, 2005)

77. K.-H. Küfer, M. Monz, A. Scherrer, P. Süss, F. Alonso, A. S. A. Sultan, Th. Bortfeld,

D. Craft, Chr. Thieke

Multicriteria optimization in intensity modulated radiotherapy planning Keywords: multicriteria optimization, extreme solutions, real-time decision making, adaptive approxima tion schemes, clustering methods, IMRT planning, reverse engineering

(51 pages, 2005)

78. S. Amstutz, H. Andrä

A new algorithm for topology optimization using a level-set method

Keywords: shape optimization, topology optimization topological sensitivity, level-set

(22 pages, 2005)

\section{N. Ettrich}

Generation of surface elevation models for urban drainage simulation

Keywords: Flooding, simulation, urban elevation models, laser scanning

(22 pages, 2005

80. H. Andrä, J. Linn, I. Matei, I. Shklyar, K. Steiner, E. Teichmann

OPTCAST - Entwicklung adäquater Struk turoptimierungsverfahren für Gießereien Technischer Bericht (KURZFASSUNG) Keywords: Topologieoptimierung, Level-Set-Methode Gießprozesssimulation, Gießtechnische Restriktionen, CAE-Kette zur Strukturoptimierung

(77 pages, 2005)
81. N. Marheineke, R. Wegener Fiber Dynamics in Turbulent Flows Part I: General Modeling Framework Keywords: fiber-fluid interaction; Cosserat rod; turbuence modeling: Kolmogorov's energy spectrum; double-velocity correlations; differentiable Gaussian fields (20 pages, 2005)

\section{Part II: Specific Taylor Drag}

Keywords: flexible fibers; $k-\varepsilon$ turbulence model; fiber-turbulence interaction scales; air drag; random Gaussian aerodynamic force; white noise; stochastic differential equations; ARMA process

(18 pages, 2005)

\section{C. H. Lampert, O. Wirjadi}

An Optimal Non-Orthogonal Separation of the Anisotropic Gaussian Convolution Filter Keywords: Anisotropic Gaussian filter, linear filtering, or entation space, $n D$ image processing, separable filters (25 pages, 2005)

83. H. Andrä, D. Stoyanov

Error indicators in the parallel finite element solver for linear elasticity DDFEM Keywords: linear elasticity, finite element method, hierarchical shape functions, domain decom-position, parallel implementation, a posteriori error estimates (21 pages, 2006)

84. M. Schröder, I. Solchenbach Optimization of Transfer Quality in Regional Public Transit

Keywords: public transit, transfer quality, quadratic assignment problem

(16 pages, 2006

85. A. Naumovich, F. J. Gaspar

On a multigrid solver for the three-dimensional Biot poroelasticity system in multilayered domains

Keywords: poroelasticity, interface problem, multigrid, operator-dependent prolongation

(11 pages, 2006)

86. S. Panda, R. Wegener, N. Marheineke Slender Body Theory for the Dynamics of Curved Viscous Fibers

Keywords: curved viscous fibers; fluid dynamics; NavierStokes equations; free boundary value problem; asymptotic expansions; slender body theory

(14 pages, 2006)

87. E. Ivanov, H. Andrä, A. Kudryavtsev Domain Decomposition Approach for Automatic Parallel Generation of Tetrahedral Grids Key words: Grid Generation, Unstructured Grid, Delau nay Triangulation, Parallel Programming, Domain Decomposition, Load Balancing

(18 pages, 2006)

88. S. Tiwari, S. Antonov, D. Hietel, J. Kuhnert, R. Wegener

A Meshfree Method for Simulations of In teractions between Fluids and Flexible Structures

Key words: Meshfree Method, FPM, Fluid Structure Interaction, Sheet of Paper, Dynamical Coupling (16 pages, 2006)

89. R. Ciegis, O. Iliev, V. Starikovicius, K. Steine Numerical Algorithms for Solving Problems of Multiphase Flows in Porous Media Keywords: nonlinear algorithms, finite-volume method software tools, porous media, flows

16 pages, 2006) 
90. D. Niedziela, O. Iliev, A. Latz

On 3D Numerical Simulations of Viscoelastic Fluids

Keywords: non-Newtonian fluids, anisotropic viscosity, integral constitutive equation

(18 pages, 2006)

\section{A. Winterfeld}

Application of general semi-infinite Programming to Lapidary Cutting Problems Keywords: large scale optimization, nonlinear programming, general semi-infinite optimization, design centering, clustering

(26 pages, 2006)

\section{J. Orlik, A. Ostrovska}

Space-Time Finite Element Approximation and Numerical Solution of Hereditary Linear Viscoelasticity Problems

Keywords: hereditary viscoelasticity; kern approximation by interpolation; space-time finite element approximation, stability and a priori estimate

(24 pages, 2006)

93. V. Rutka, A. Wiegmann, H. Andrä EJIIM for Calculation of effective Elastic Moduli in 3D Linear Elasticity

Keywords: Elliptic PDE, linear elasticity, irregular domain, finite differences, fast solvers, effective elastic moduli

(24 pages, 2006)

\section{A. Wiegmann, A. Zemitis}

EJ-HEAT: A Fast Explicit Jump Harmonic Averaging Solver for the Effective Heat Conductivity of Composite Materials Keywords: Stationary heat equation, effective thermal conductivity, explicit jump, discontinuous coefficients, virtual material design, microstructure simulation, EJ-HEAT

(21 pages, 2006)

\section{A. Naumovich}

On a finite volume discretization of the three-dimensional Biot poroelasticity system in multilayered domains

Keywords: Biot poroelasticity system, interface problems, finite volume discretization, finite difference method (21 pages, 2006)

\section{M. Krekel, J. Wenzel}

A unified approach to Credit Default Swaption and Constant Maturity Credit Default Swap valuation

Keywords: LIBOR market model, credit risk, Credit Default Swaption, Constant Maturity Credit Default Swapmethod

(43 pages, 2006)

\section{A. Dreyer}

\section{Interval Methods for Analog Circiuts}

Keywords: interval arithmetic, analog circuits, tolerance analysis, parametric linear systems, frequency response, symbolic analysis, CAD, computer algebra

(36 pages, 2006)

98. N. Weigel, S. Weihe, G. Bitsch, K. Dreßler Usage of Simulation for Design and Optimization of Testing

Keywords: Vehicle test rigs, MBS, control, hydraulics,

testing philosophy

(14 pages, 2006)

99. H. Lang, G. Bitsch, K. Dreßler, M. Speckert Comparison of the solutions of the elastic and elastoplastic boundary value problems
Keywords: Elastic BVP, elastoplastic BVP, variational inequalities, rate-independency, hysteresis, linear kinematic hardening, stop- and play-operator

(21 pages, 2006)

100. M. Speckert, K. Dreßler, H. Mauch MBS Simulation of a hexapod based suspension test rig

Keywords: Test rig, MBS simulation, suspension,

hydraulics, controlling, design optimization

(12 pages, 2006)

101. S. Azizi Sultan, K.-H. Küfer

A dynamic algorithm for beam orientations in multicriteria IMRT planning

Keywords: radiotherapy planning, beam orientation optimization, dynamic approach, evolutionary algorithm, global optimization

(14 pages, 2006)

102. T. Götz, A. Klar, N. Marheineke, R. Wegener A Stochastic Model for the Fiber Lay-down Process in the Nonwoven Production Keywords: fiber dynamics, stochastic Hamiltonian system, stochastic averaging

(17 pages, 2006)

103. Ph. Süss, K.-H. Küfer

Balancing control and simplicity: a variable aggregation method in intensity modulated radiation therapy planning

Keywords: IMRT planning, variable aggregation, clustering methods

(22 pages, 2006)

104. A. Beaudry, G. Laporte, T. Melo, S. Nickel Dynamic transportation of patients in hospitals

Keywords: in-house hospital transportation, dial-a-ride, dynamic mode, tabu search

(37 pages, 2006)

105. Th. Hanne

Applying multiobjective evolutionary algorithms in industrial projects

Keywords: multiobjective evolutionary algorithms, discrete optimization, continuous optimization, electronic circuit design, semi-infinite programming, scheduling (18 pages, 2006)

106. J. Franke, S. Halim

Wild bootstrap tests for comparing signals and images

Keywords: wild bootstrap test, texture classification, textile quality control, defect detection, kernel estimate, nonparametric regression

(13 pages, 2007)

107. Z. Drezner, S. Nickel

Solving the ordered one-median problem in the plane

Keywords: planar location, global optimization, ordered median, big triangle small triangle method, bounds, numerical experiments

(21 pages, 2007)

108. Th. Götz, A. Klar, A. Unterreiter, R. Wegener

Numerical evidance for the non-existing of solutions of the equations desribing rotational fiber spinning

Keywords: rotational fiber spinning, viscous fibers, boundary value problem, existence of solutions (11 pages, 2007)
109. Ph. Süss, K.-H. Küfer

Smooth intensity maps and the BortfeldBoyer sequencer

Keywords: probabilistic analysis, intensity modulated radiotherapy treatment (IMRT), IMRT plan application step-and-shoot sequencing

(8 pages, 2007)

110. E. Ivanov, O. Gluchshenko, H. Andrä, A. Kudryavtsev

Parallel software tool for decomposing and meshing of $3 d$ structures

Keywords: a-priori domain decomposition, unstructured grid, Delaunay mesh generation

(14 pages, 2007)

111. O. lliev, R. Lazarov, J. Willems Numerical study of two-grid preconditioners for 1d elliptic problems with highly oscillating discontinuous coefficients Keywords: two-grid algorithm, oscillating coefficients, preconditioner

(20 pages, 2007)

112. L. Bonilla, T. Götz, A. Klar, N. Marheineke, R. Wegener

Hydrodynamic limit of the Fokker-Planckequation describing fiber lay-down processes

Keywords: stochastic dierential equations, FokkerPlanck equation, asymptotic expansion, Ornstein-

Uhlenbeck process

(17 pages, 2007)

\section{S. Rief}

Modeling and simulation of the pressing section of a paper machine

Keywords: paper machine, computational fluid dynamics, porous media

(41 pages, 2007)

114. R. Ciegis, O. Iliev, Z. Lakdawala

On parallel numerical algorithms for simulating industrial filtration problems

Keywords: Navier-Stokes-Brinkmann equations, finite volume discretization method, SIMPLE, parallel computing, data decomposition method

(24 pages, 2007)

\section{N. Marheineke, R. Wegener}

Dynamics of curved viscous fibers with surface tension

Keywords: Slender body theory, curved viscous bers with surface tension, free boundary value problem (25 pages, 2007)

116. S. Feth, J. Franke, M. Speckert Resampling-Methoden zur mse-Korrektur und Anwendungen in der Betriebsfestigkeit Keywords: Weibull, Bootstrap, Maximum-Likelihood, Betriebsfestigkeit

(16 pages, 2007)

117. H. Knaf

Kernel Fisher discriminant functions - a concise and rigorous introduction

Keywords: wild bootstrap test, texture classification, textile quality control, defect detection, kernel estimate, nonparametric regression

(30 pages, 2007)

118. O. Iliev, I. Rybak

On numerical upscaling for flows in heterogeneous porous media 
Keywords: numerical upscaling heterogeneous porous media, single phase flow, Darcy's law, multiscale problem, effective permeability, multipoint flux approximation, anisotropy

(17 pages, 2007)

\section{O. Iliev, I. Rybak}

On approximation property of multipoint flux approximation method

Keywords: Multipoint flux approximation, finite volume method, elliptic equation, discontinuous tensor coeffi-

cients, anisotropy

(15 pages, 2007)

120. O. Iliev, I. Rybak, J. Willems

On upscaling heat conductivity for a class of industrial problems

Keywords: Multiscale problems, effective heat conductivity, numerical upscaling, domain decomposition

(21 pages, 2007)

121. R. Ewing, O. Iliev, R. Lazarov, I. Rybak On two-level preconditioners for flow in porous media

Keywords: Multiscale problem, Darcy's law, single phase flow, anisotropic heterogeneous porous media, numerical upscaling, multigrid, domain decomposition, efficient preconditioner

(18 pages, 2007)

122. M. Brickenstein, A. Dreyer

POLYBORI: A Gröbner basis framework for Boolean polynomials

Keywords: Gröbner basis, formal verification, Boolean polynomials, algebraic cryptoanalysis, satisfiability

(23 pages, 2007)

\section{O. Wirjad}

Survey of $3 d$ image segmentation methods Keywords: image processing, 3d, image segmentation, binarization

(20 pages, 2007)

\section{S. Zeytun, A. Gupta}

A Comparative Study of the Vasicek and the CIR Model of the Short Rate

Keywords: interest rates, Vasicek model, CIR-model,

calibration, parameter estimation

(17 pages, 2007)

\section{G. Hanselmann, A. Sarishvili}

Heterogeneous redundancy in software quality prediction using a hybrid Bayesian approach

Keywords: reliability prediction, fault prediction, nonhomogeneous poisson process, Bayesian model averaging

(17 pages, 2007)

126. V. Maag, M. Berger, A. Winterfeld, K.-H Küfer

A novel non-linear approach to minimal area rectangular packing

Keywords: rectangular packing, non-overlapping constraints, non-linear optimization, regularization, relaxation

(18 pages, 2007)

127. M. Monz, K.-H. Küfer, T. Bortfeld, C. Thieke Pareto navigation - systematic multi-criteria-based IMRT treatment plan determination

Keywords: convex, interactive multi-objective optimization, intensity modulated radiotherapy planning

(15 pages, 2007)
128. M. Krause A Scherrer

On the role of modeling parameters in IMRT plan optimization

Keywords: intensity-modulated radiotherapy (IMRT) inverse IMRT planning, convex optimization, sensitivity analysis, elasticity, modeling parameters, equivalent uniform dose (EUD)

(18 pages, 2007)

\section{A. Wiegmann}

Computation of the permeability of porous materials from their microstructure by FFFStokes

Keywords: permeability, numerical homogenization

fast Stokes solver

(24 pages, 2007)

130. T. Melo, S. Nickel, F. Saldanha da Gama Facility Location and Supply Chain Management - A comprehensive review

Keywords: facility location, supply chain management network design

(54 pages, 2007)

131. T. Hanne, T. Melo, S. Nickel

Bringing robustness to patient flow management through optimized patient transports in hospitals

Keywords: Dial-a-Ride problem, online problem, case study, tabu search, hospital logistics

(23 pages, 2007)

132. R. Ewing, O. Iliev, R. Lazarov, I. Rybak, J. Willems

An efficient approach for upscaling properties of composite materials with high contrast of coefficients

Keywords: effective heat conductivity, permeability of fractured porous media, numerical upscaling, fibrous insulation materials, metal foams

(16 pages, 2008)

133. S. Gelareh, S. Nickel

New approaches to hub location problems in public transport planning Keywords: integer programming, hub location, transportation, decomposition, heuristic

(25 pages, 2008)

134. G. Thömmes, J. Becker, M. Junk, A. K. Vaikuntam, D. Kehrwald, A. Klar, K. Steiner, A. Wiegmann

A Lattice Boltzmann Method for immiscible multiphase flow simulations using the Level Set Method

Keywords: Lattice Boltzmann method, Level Set method, free surface, multiphase flow (28 pages, 2008

\section{J. Orlik}

Homogenization in elasto-plasticity

Keywords: multiscale structures, asymptotic homogenization, nonlinear energy

(40 pages, 2008)

136. J. Almquist, H. Schmidt, P. Lang, J. Deitmer, M. Jirstrand, D. Prätzel-Wolters, H. Becker

Determination of interaction between MCT1 and CAII via a mathematical and physiological approach

Keywords: mathematical modeling; model reduction: electrophysiology; $\mathrm{pH}$-sensitive microelectrodes; proton antenna

(20 pages, 2008)
137. E. Savenkov, H. Andrä, O. Iliev

An analysis of one regularization approach for solution of pure Neumann problem Keywords: pure Neumann problem, elasticity, regularization, finite element method, condition number (27 pages, 2008)

138. O. Berman, J. Kalcsics, D. Krass, S. Nickel The ordered gradual covering location problem on a network

Keywords: gradual covering, ordered median function, network location

(32 pages, 2008)

139. S. Gelareh, S. Nickel

Multi-period public transport design: A novel model and solution approaches Keywords: Integer programming, hub location, public transport, multi-period planning, heuristics (31 pages, 2008)

140. T. Melo, S. Nickel, F. Saldanha-da-Gama Network design decisions in supply chain planning

Keywords: supply chain design, integer programming models, location models, heuristics

(20 pages, 2008)

141. C. Lautensack, A. Särkkä, J. Freitag K. Schladitz

Anisotropy analysis of pressed point processes

Keywords: estimation of compression, isotropy test nearest neighbour distance, orientation analysis, polar ice, Ripley's K function

(35 pages, 2008)

142. O. Iliev, R. Lazarov, J. Willems

A Graph-Laplacian approach for calculating the effective thermal conductivity of complicated fiber geometries

Keywords: graph laplacian, effective heat conductivity, numerical upscaling, fibrous materials

(14 pages, 2008)

143. J. Linn, T. Stephan, J. Carlsson, R. Bohlin Fast simulation of quasistatic rod deformations for VR applications

Keywords: quasistatic deformations, geometrically exact rod models, variational formulation, energy minimization, finite differences, nonlinear conjugate gradients

(7 pages, 2008)

\section{J. Linn, T. Stephan}

Simulation of quasistatic deformations using discrete rod models

Keywords: quasistatic deformations, geometrically exact rod models, variational formulation, energy minimization, finite differences, nonlinear conjugate gradients

(9 pages, 2008

145. J. Marburger, N. Marheineke, R. Pinnau Adjoint based optimal control using meshless discretizations

Keywords: Mesh-less methods, particle methods, Eulerian-Lagrangian formulation, optimization strategies, adjoint method, hyperbolic equations

(14 pages, 2008

\section{S. Desmettre, J. Gould, A. Szimayer}

Own-company stockholding and work effort preferences of an unconstrained executive Keywords: optimal portfolio choice, executive compensation

(33 pages, 2008) 
147. M. Berger, M. Schröder, K.-H. Küfer A constraint programming approach for the two-dimensional rectangular packing problem with orthogonal orientations

Keywords: rectangular packing, orthogonal orientations non-overlapping constraints, constraint propagation

(13 pages, 2008)

148. K. Schladitz, C. Redenbach, T. Sych, M. Godehardt

Microstructural characterisation of open foams using $3 d$ images

Keywords: virtual material design, image analysis, open foams

(30 pages, 2008)

149. E. Fernández, J. Kalcsics, S. Nickel, R. Ríos-Mercado

A novel territory design model arising in the implementation of the WEEE-Directive Keywords: heuristics, optimization, logistics, recycling (28 pages, 2008)

150. H. Lang, J. Linn

Lagrangian field theory in space-time for geometrically exact Cosserat rods Keywords: Cosserat rods, geometrically exact rods, small strain, large deformation, deformable bodies, Lagrangian field theory, variational calculus (19 pages, 2009)

151. K. Dreßler, M. Speckert, R. Müller, Ch. Weber

Customer loads correlation in truck engineering

Keywords: Customer distribution, safety critical components, quantile estimation, Monte-Carlo methods (11 pages, 2009)

152. H. Lang, K. Dreßler

An improved multiaxial stress-strain correction model for elastic FE postprocessing Keywords: Jiang's model of elastoplasticity, stress-strain correction, parameter identification, automatic differentiation, least-squares optimization, Coleman-Li algorithm

(6 pages, 2009)

153. J. Kalcsics, S. Nickel, M. Schröder A generic geometric approach to territory design and districting

Keywords: Territory design, districting, combinatorial optimization, heuristics, computational geometry (32 pages, 2009)

154. Th. Fütterer, A. Klar, R. Wegener An energy conserving numerical scheme for the dynamics of hyperelastic rods

Keywords: Cosserat rod, hyperealstic, energy conservation, finite differences

(16 pages, 2009)

155. A. Wiegmann, L. Cheng, E. Glatt, O. Iliev, S. Rief

Design of pleated filters by computer simulations

Keywords: Solid-gas separation, solid-liquid separation, pleated filter, design, simulation

(21 pages, 2009)

156. A. Klar, N. Marheineke, R. Wegener Hierarchy of mathematical models for production processes of technical textiles
Keywords: Fiber-fluid interaction, slender-body theory, turbulence modeling, model reduction, stochastic differential equations, Fokker-Planck equation, asymptotic expansions, parameter identification

(21 pages, 2009)

157. E. Glatt, S. Rief, A. Wiegmann, M. Knefel, E. Wegenke

Structure and pressure drop of real and virtual metal wire meshes

Keywords: metal wire mesh, structure simulation, model calibration, CFD simulation, pressure loss (7 pages, 2009)

\section{S. Kruse, M. Mülle}

Pricing American call options under the assumption of stochastic dividends - An application of the Korn-Rogers model Keywords: option pricing, American options, dividends, dividend discount model, Black-Scholes model (22 pages, 2009)

159. H. Lang, J. Linn, M. Arnold

Multibody dynamics simulation of geometrically exact Cosserat rods

Keywords: flexible multibody dynamics, large deformations, finite rotations, constrained mechanical systems, structural dynamics

(20 pages, 2009)

160. P. Jung, S. Leyendecker, J. Linn, M. Ortiz Discrete Lagrangian mechanics and geometrically exact Cosserat rods

Keywords: special Cosserat rods, Lagrangian mechanics, Noether's theorem, discrete mechanics, frame-indifference, holonomic constraints

(14 pages, 2009)

161. M. Burger, K. Dreßler, A. Marquardt, M. Speckert

Calculating invariant loads for system simulation in vehicle engineering

Keywords: iterative learning control, optimal control

theory, differential algebraic equations (DAEs)

(18 pages, 2009)

162. M. Speckert, N. Ruf, K. Dreßler Undesired drift of multibody models excited by measured accelerations or forces Keywords: multibody simulation, full vehicle model, force-based simulation, drift due to noise (19 pages, 2009)

163. A. Streit, K. Dreßler, M. Speckert, J. Lichter, T. Zenner, P. Bach

Anwendung statistischer Methoden zur Erstellung von Nutzungsprofilen für die Auslegung von Mobilbaggern

Keywords: Nutzungsvielfalt, Kundenbeanspruchung, Bemessungsgrundlagen

(13 pages, 2009)

164. I. Correia, S. Nickel, F. Saldanha-da-Gama The capacitated single-allocation hub location problem revisited: A note on a classical formulation

Keywords: Capacitated Hub Location, MIP formulations (10 pages, 2009)

165. F. Yaneva, T. Grebe, A. Scherrer

An alternative view on global radiotherapy optimization problems

Keywords: radiotherapy planning, path-connected sublevelsets, modified gradient projection method, improving and feasible directions

(14 pages, 2009)
166. J. I. Serna, M. Monz, K.-H. Küfer, C. Thieke Trade-off bounds and their effect in multicriteria IMRT planning

Keywords: trade-off bounds, multi-criteria optimization, IMRT, Pareto surface

(15 pages, 2009)

167. W. Arne, N. Marheineke, A. Meister, R. Wegener

Numerical analysis of Cosserat rod and string models for viscous jets in rotational spinning processes

Keywords: Rotational spinning process, curved viscous fibers, asymptotic Cosserat models, boundary value problem, existence of numerical solutions

(18 pages, 2009)

168. T. Melo, S. Nickel, F. Saldanha-da-Gama An LP-rounding heuristic to solve a multiperiod facility relocation problem

Keywords: supply chain design, heuristic, linear programming, rounding

(37 pages, 2009)

169. I. Correia, S. Nickel, F. Saldanha-da-Gama Single-allocation hub location problems with capacity choices

Keywords: hub location, capacity decisions, MILP formulations

(27 pages, 2009)

170. S. Acar, K. Natcheva-Acar

A guide on the implementation of the

Heath-Jarrow-Morton Two-Factor Gaussian

Short Rate Model (HJM-G2++)

Keywords: short rate model, two factor Gaussian,

G2++, option pricing, calibration

(30 pages, 2009)

171. A. Szimayer, G. Dimitroff, S. Lorenz A parsimonious multi-asset Heston model: calibration and derivative pricing

Keywords: Heston model, multi-asset, option pricing, calibration, correlation

(28 pages, 2009)

\section{N. Marheineke, R. Wegener}

Modeling and validation of a stochastic drag for fibers in turbulent flows

Keywords: fiber-fluid interactions, long slender fibers, turbulence modelling, aerodynamic drag, dimensional analysis, data interpolation, stochastic partial differential algebraic equation, numerical simulations, experimental validations

(19 pages, 2009)

173. S. Nickel, M. Schröder, J. Steeg Planning for home health care services Keywords: home health care, route planning, metaheuristics, constraint programming

(23 pages, 2009)

174. G. Dimitroff, A. Szimayer, A. Wagner Quanto option pricing in the parsimonious Heston model

Keywords: Heston model, multi asset, quanto options, option pricing

(14 pages, 2009) 174. G. Dimitroff, A. Szimayer, A. Wagner

175. S. Herkt, K. Dreßler, R. Pinnau

Model reduction of nonlinear problems in structural mechanics

Keywords: flexible bodies, FEM, nonlinear model reduction, POD

(13 pages, 2009) 
176. M. K. Ahmad, S. Didas, J. Iqbal

Using the Sharp Operator for edge detection and nonlinear diffusion

Keywords: maximal function, sharp function, image processing, edge detection, nonlinear diffusion

(17 pages, 2009)

177. M. Speckert, N. Ruf, K. Dreßler, R. Müller, C. Weber, S. Weihe

Ein neuer Ansatz zur Ermittlung von Erprobungslasten für sicherheitsrelevante Bauteile

Keywords: sicherheitsrelevante Bauteile, Kundenbeanspruchung, Festigkeitsverteilung, Ausfallwahrscheinlichkeit, Konfidenz, statistische Unsicherheit, Sicher-

heitsfaktoren

(16 pages, 2009)

178. J. Jegorovs

Wave based method: new applicability areas Keywords: Elliptic boundary value problems, inhomogeneous Helmholtz type differential equations in bounded domains, numerical methods, wave based method, uniform $B$-splines

(10 pages, 2009)

179. H. Lang, M. Arnold

Numerical aspects in the dynamic simulation of geometrically exact rods Keywords: Kirchhoff and Cosserat rods, geometrically exact rods, deformable bodies, multibody dynamics, artial differential algebraic equations, method of lines, time integration

(21 pages, 2009)

180. H. Lang

Comparison of quaternionic and rotationfree null space formalisms for multibody dynamics

Keywords: Parametrisation of rotations, differentialalgebraic equations, multibody dynamics, constrained mechanical systems, Lagrangian mechanics

(40 pages, 2010)

181. S. Nickel, F. Saldanha-da-Gama, H.-P. Ziegler Stochastic programming approaches for risk aware supply chain network design problems Keywords: Supply Chain Management, multi-stage stochastic programming, financial decisions, risk (37 pages, 2010)

182. P. Ruckdeschel, N. Horbenko

Robustness properties of estimators in generalized Pareto Models

Keywords: global robustness, local robustness, finite

sample breakdown point, generalized Pareto distribution

(58 pages, 2010)

183. P. Jung, S. Leyendecker, J. Linn, M. Ortiz

A discrete mechanics approach to Cosserat rod theory - Part 1: static equilibria

Keywords: Special Cosserat rods; Lagrangian mechanics; Noether's theorem; discrete mechanics; frameindifference; holonomic constraints; variational formulation

(35 pages, 2010)

184. R. Eymard, G. Printsypar

A proof of convergence of a finite volume scheme for modified steady Richards' equation describing transport processes in the pressing section of a paper machine Keywords: flow in porous media, steady Richards' equation, finite volume methods, convergence of approximate solution

(14 pages, 2010)

185. P. Ruckdeschel

Optimally Robust Kalman Filtering

Keywords: robustness, Kalman Filter, innovation outlier, additive outlier

(42 pages, 2010)

186. S. Repke, N. Marheineke, R. Pinnau On adjoint-based optimization of a free surface Stokes flow

Keywords: film casting process, thin films, free surface

Stokes flow, optimal control, Lagrange formalism

(13 pages, 2010)

187. O. Iliev, R. Lazarov, J. Willems

Variational multiscale Finite Element Method for flows in highly porous media

Keywords: numerical upscaling, flow in heterogeneous porous media, Brinkman equations, Darcy's law, subgrid approximation, discontinuous Galerkin mixed FEM (21 pages, 2010)

\section{S. Desmettre, A. Szimayer}

Work effort, consumption, and portfolio selection: When the occupational choice matters

Keywords: portfolio choice, work effort, consumption, occupational choice

(34 pages, 2010)

189. O. Iliev, Z. Lakdawala, V. Starikovicius

On a numerical subgrid upscaling algorithm for Stokes-Brinkman equations

Keywords: Stokes-Brinkman equations, subgrid

approach, multiscale problems, numerical upscaling (27 pages, 2010)

190. A. Latz, J. Zausch, O. Iliev

Modeling of species and charge transport in Li-Ion Batteries based on non-equilibrium thermodynamics

Keywords: lithium-ion battery, battery modeling, elec trochemical simulation, concentrated electrolyte, ion transpor

(8 pages, 2010)

191. P. Popov, Y. Vutov, S. Margenov, O. Iliev Finite volume discretization of equations describing nonlinear diffusion in Li-lon batteries

Keywords: nonlinear diffusion, finite volume discretization, Newton method, Li-lon batteries

(9 pages, 2010)

192. W. Arne, N. Marheineke, R. Wegener Asymptotic transition from Cosserat rod to string models for curved viscous inertial jets

Keywords: rotational spinning processes; inertial and viscous-inertial fiber regimes; asymptotic limits; slenderbody theory; boundary value problems

(23 pages, 2010)

193. L. Engelhardt, M. Burger, G. Bitsch Real-time simulation of multibody-systems for on-board applications

Keywords: multibody system simulation, real-time simulation, on-board simulation, Rosenbrock methods (10 pages, 2010)

194. M. Burger, M. Speckert, K. Dreßler Optimal control methods for the calculation of invariant excitation signals for multibody systems

Keywords: optimal control, optimization, mbs simulation, invariant excitation

(9 pages, 2010)
195. A Latz, J. Zausch

Thermodynamic consistent transport theory of Li-lon batteries

Keywords: Li-lon batteries, nonequilibrium thermodynamics, thermal transport, modeling

(18 pages, 2010)

\section{S. Desmettre}

Optimal investment for executive stockholders with exponential utility Keywords: portfolio choice, executive stockholder, work effort, exponential utility

(24 pages, 2010)

197. W. Arne, N. Marheineke, J. Schnebele, R. Wegener

Fluid-fiber-interactions in rotational spinning process of glass wool production Keywords: Rotational spinning process, viscous thermal jets, fluid-fiber-interactions, two-way coupling, slenderbody theory, Cosserat rods, drag models, boundary value problem, continuation method

(20 pages, 2010)

198. A. Klar, J. Maringer, R. Wegener A 3d model for fiber lay-down in nonwoven production processes

Keywords: fiber dynamics, Fokker-Planck equations, diffusion limits

(15 pages, 2010)

Status quo: December 2010 